\title{
LA ATENUACIÓN DE LAS GUÍAS DE ONDAS CIRCULARES CON DIELÉCTRICO DE AIRE
}

\author{
LOSSES IN AIR-FILLED CIRCULAR WAVEGUIDES
}

Víctor Cruz Ornetta', Harold Cruz Salas²,
Vanessa Vásquez Garro ${ }^{2}$

RECEPCIÓN: 10 DE OCTUBRE DEL 2019

ACEPTACIÓN: 06 DE NOVIEMBRE DEL 2019

\section{RESUMEN}

Las guías de ondas son elementos importantes de los sistemas de transmisión por microondas, pero también son poco conocidas, especialmente, en el caso de las atenuaciones de las guías de ondas circulares. Por ello, el objetivo de este artículo es presentar, de manera clara y detallada, los cálculos para la obtención de las atenuaciones en las guías de onda circulares, de tal manera que contribuya a la sólida formación de alumnos de las carreras involucradas en estos temas. Para el desarrollo de este estudio, se recurrió a los libros de texto de campos electromagnéticos más reconocidos y a algunos papers. Asimismo, los cálculos se realizaron mediante Excel versión 1909 de Microsoft Office 365 Pro Plus. Como resultado, se encontró que las atenuaciones provocadas por estas guías son de pequeña magnitud. Por otro lado, se mostró que las fórmulas utilizadas son las correctas, ya que los resultados coinciden, casi en su totalidad, con aquellos alcanzados por científicos reconocidos a nivel mundial.

Palabras clave: guías de ondas; guías de ondas circulares; guías de ondas cilíndricas; atenuaciones de guías de ondas; pérdidas de guías de ondas.

\begin{abstract}
Waveguides are important components of microwave transmission systems, but they are mostly unknown specially about circular waveguide losses. That is the reason why the objective of this study is to present very well detailed the computations to obtain the losses for circular microwaves to contribute to a better formation of the students of the careers involved in this subject. To develop this study we have used the most classic books for electromagnetic fields and some papers and the computations were carried out with the software Excel versión 1909 de Microsoft Office 365 Pro Plus. As a result it was found that losses of circular waveguides are small and our computations gave our similar outputs as the computations done by very well known scientists.
\end{abstract}

Keywords: waveguides; circular waveguides; cilidrical waveguides; waveguide attenuations; waveguide losses.

$1 \quad$ Universidad Ricardo Palma. Facultad de Ingeniería. Email: vcruzor@gmail.com

2 Universidad Nacional Mayor de San Marcos. Email harold.cruz@unmsm.edu.pe, vaneevasquezg@gmail.com 


\section{INTRODUCCIÓN}

Las guías de ondas circulares ocasionalmente son utilizadas como una alternativa a las guías de onda rectangulares. Estas guías son construidas con base en un conductor único cilíndrico.

En los libros de texto normalmente se presentan las ecuaciones de atenuación, pero no las curvas de atenuación $[1,2,3,6,7]$. Por el contrario, en algunos artículos científicos, se presentan las curvas de atenuación $[4,5]$; sin embargo, no se presentan los cálculos detallados. Por esa razón, el alumno no sabe cómo aplicarlas e incurre en serios errores, debido a que no son claras las unidades que utilizan. Es por ello que el objetivo de este artículo es presentar de manera clara y detallada los cálculos para la obtención de las pérdidas en las guías de onda circulares, de tal manera que contribuya a la sólida formación de alumnos de las carreras involucradas en estos temas.

\section{MARCO TEÓRICO}

De acuerdo a la literatura consultada para el artículo, la atenuación en las guías de ondas circulares con dieléctrico de aire está dada, principalmente, por las pérdidas en el conductor. Por ello, deben despreciarse las pérdidas en el dieléctrico.

En este caso, las pérdidas para los modos transversales eléctricos, de manera general, están expresadas de la siguiente manera:

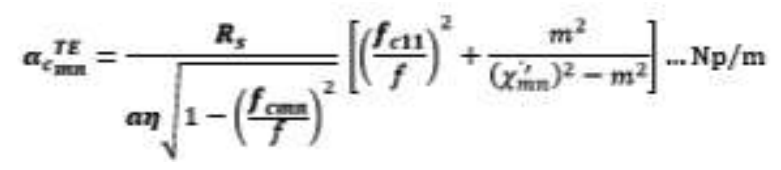

Particularmente, para los modos transversales eléctricos TE01, TE02, TE11 y TE21, están expresadas las siguientes ecuaciones:

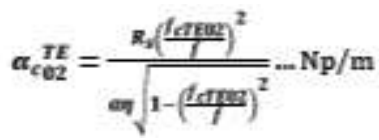

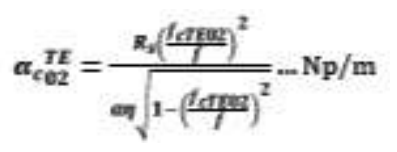

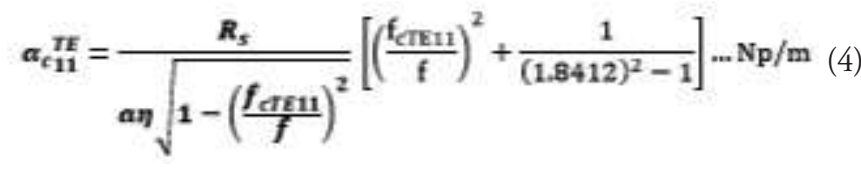

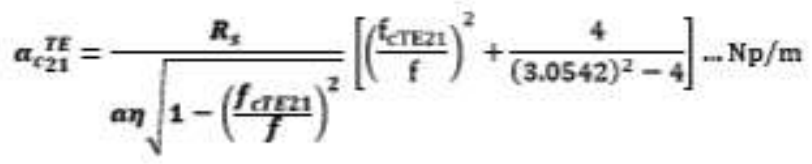


En el caso de los modos transversales magnéticos, la atenuación, de manera general, está expresada por:

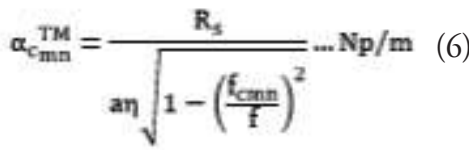

En los casos particulares de los modos TM01 y TM11, las pérdidas se expresan por las ecuaciones siguientes:

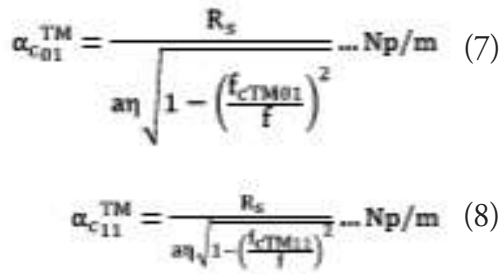

Dado que la solución de las guías de ondas circulares está dada en términos de las funciones de Bessel, en las tablas 1 y 2 presentamos los ceros de la función de Bessel y su primera derivada.

\begin{tabular}{rrrrrrrrrrrrr}
\hline & $m=0$ & $m=1$ & $m=2$ & $m=3$ & $m=4$ & $m=5$ & $m=6$ & $m=7$ & $m=8$ & $m=9$ & $m=10$ & $m=11$ \\
\hline$n=1$ & 2,405 & 3,832 & 5,136 & 6,380 & 7,588 & 8,772 & 9,936 & 11,086 & 12,225 & 13,354 & 14,476 & 12,826 \\
$n=2$ & 5,520 & 7,106 & 8,417 & 9,761 & 11,065 & 12,339 & 13,589 & 14,821 & 16,038 & 17,241 & 18,434 & 19,616 \\
$n=3$ & 8,654 & 10,174 & 11,620 & 13,015 & 14,373 & 15,700 & 17,004 & 18,288 & 19,555 & 20,807 & 22,047 & 23,276 \\
$n=4$ & 11,792 & 13,324 & 14,796 & 16,224 & 17,616 & 18,980 & 20,321 & 21,642 & 22,945 & 24,234 & 25,510 & 26,773 \\
$n=5$ & 14,931 & 16,471 & 17,960 & 19,409 & 20,827 & 22,218 & 23,586 & 24,935 & 26,267 & 27,584 & 28,887 & 80,179 \\
\hline
\end{tabular}

Tabla 1. Ceros $X_{m n}$ de la función de Bessel $J_{m}\left(X_{m n}\right)=0(n=1,2,3 \ldots)$.

Elaboración propia.

\begin{tabular}{rrrrrrrrrrrrrr}
\hline & $m=0$ & $m=1$ & $m=2$ & $m=3$ & $m=4$ & $m=5$ & $m=6$ & $m=7$ & $m=8$ & $m=9$ & $m=10$ & $m=11$ \\
\hline$n=1$ & 3,832 & 1,841 & 3,054 & 4,201 & 5,318 & 6,416 & 7,501 & 8,578 & 9,647 & 10,711 & 11,771 & 12,826 \\
$n=2$ & 7,016 & 5,332 & 6,706 & 8,015 & 9,282 & 10,520 & 11,735 & 12,932 & 14,116 & 15,287 & 16,448 & 17,600 \\
$n=3$ & 10,174 & 8,536 & 9,970 & 11,346 & 12,682 & 13,987 & 15,268 & 16,529 & 17,774 & 19,005 & 20,223 & 21,431 \\
$n=4$ & 13,324 & 11,706 & 13,170 & 14,586 & 15,964 & 17,313 & 18,638 & 19,942 & 21,229 & 22,501 & 23,761 & 25,009 \\
$n=5$ & 16,471 & 14,864 & 16,348 & 17,789 & 19,196 & 20,576 & 21,932 & 23,268 & 24,587 & 25,891 & 27,182 & 28,461 \\
\hline
\end{tabular}

Tabla 2. Ceros $X_{m n}^{\prime}$ de la derivada $J_{m}^{\prime}\left(X_{m n}^{\prime}\right)=0(n=1,2,3 \ldots)$ de la función de Bessel $J_{m}(X)$.

Elaboración propia. 


\section{MATERIALES Y MÉTODOS}

Para la realización de este estudio, se revisaron los libros de texto de campos electromagnéticos más reconocidos y algunos papers, y los cálculos se realizaron mediante Excel versión 1909 de Microsoft Office 365 Pro Plus.

\section{RESULTADOS}

Desde la tabla 3 hasta la 16, se presentan los cálculos de las atenuaciones para guías de ondas circulares de 1.5 y $3 \mathrm{~cm}$ de diámetro para el modo TE01, TE02, TE11, TE21, TM01 y TM11. De la misma manera, en los cuadros 1 al 6 y 8 al 13, se presentan las gráficas de las atenuaciones individuales para los modos TE01, TE02, TE11, TE21, TM01 y TM11 de las guías de ondas circulares de 1.5 y 3 $\mathrm{cm}$, mientras que los cuadros 7 y 14 muestran la comparación de atenuaciones para las guías de ondas circulares de 1.5 y $3 \mathrm{~cm}$, respectivamente.

\begin{tabular}{|c|c|c|c|c|c|c|}
\hline & $\begin{array}{c}\mathrm{f} \\
(\mathrm{GHz})\end{array}$ & $\begin{array}{c}\mathrm{R}_{\mathrm{s}} \\
\left(\mathrm{Ohmios} / \mathrm{m}^{2}\right)\end{array}$ & $\begin{array}{c}\mathrm{f}_{\text {cTE01 }} \\
(\mathrm{GHz})\end{array}$ & $(\mathrm{Np} / \mathrm{m})$ & $\begin{array}{c}\alpha \\
(\mathrm{dB} / \mathrm{m})\end{array}$ & $(\mathrm{dB} / 1000 \mathrm{~m})$ \\
\hline $1,005 f_{\text {сTE } 01}$ & 12,26 & 0,03 & 12,20 & 0,0512 & 0,4448 & 444,7653 \\
\hline $1,010 f_{\text {СTE } 01}$ & 12,32 & 0,03 & 12,20 & 0,0361 & 0,3133 & 313,3267 \\
\hline $1,100 f_{\text {стЕ } 01}$ & 13,42 & 0,03 & 12,20 & 0,0107 & 0,0929 & 92,8860 \\
\hline $3,000 \mathrm{f}_{\text {CTE } 11}$ & 17,58 & 0,03 & 12,20 & 0,0041 & 0,0000 & 35,8132 \\
\hline $4,250 \mathrm{f}_{\text {CTE } 11}$ & 24,91 & 0,04 & 12,20 & 0,0020 & 0,0175 & 17,5452 \\
\hline $5,500 \mathrm{f}_{\mathrm{CTE} 11}$ & 32,23 & 0,05 & 12,20 & 0,0013 & 0,0112 & 11,2259 \\
\hline $6,750 \mathrm{f}_{\text {CTE } 11}$ & 39,56 & 0,05 & 12,20 & 0,0009 & 0,0080 & 8,0342 \\
\hline $8,000 \mathrm{f}_{\text {CTE } 11}$ & 46,89 & 0,06 & 12,20 & 0,0007 & 0,0061 & 6,1347 \\
\hline $9,250 f_{\text {CTE } 11}$ & 54,21 & 0,06 & 12,20 & 0,0006 & 0,0049 & 4,8896 \\
\hline $10,500 \mathrm{f}_{\text {СTE } 11}$ & 61,54 & 0,07 & 12,20 & 0,0005 & 0,0040 & 4,0191 \\
\hline $11,750 \mathrm{f}_{\text {CTE11 }}$ & 68,86 & 0,07 & 12,20 & 0,0004 & 0,0034 & 3,3812 \\
\hline $13,000 \mathrm{f}_{\mathrm{CTE} 11}$ & 76,19 & 0,07 & 12,20 & 0,0003 & 0,0029 & 2,8969 \\
\hline $14,250 \mathrm{f}_{\text {CTE11 }}$ & 83,52 & 0,08 & 12,20 & 0,0003 & 0,0025 & 2,5187 \\
\hline $15,500 \mathrm{f}_{\text {CTE11 }}$ & 90,84 & 0,08 & 12,20 & 0,0003 & 0,0022 & 2,2165 \\
\hline $16,750 \mathrm{f}_{\mathrm{CTE} 11}$ & 98,17 & 0,08 & 12,20 & 0,0002 & 0,0020 & 1,9704 \\
\hline $18,000 \mathrm{f}_{\text {CTE } 11}$ & 105,49 & 0,09 & 12,20 & 0,0002 & 0,0018 & 1,7669 \\
\hline $19,250 \mathrm{f}_{\mathrm{CTE} 11}$ & 112,82 & 0,09 & 12,20 & 0,0002 & 0,0016 & 1,5963 \\
\hline $20,500 \mathrm{f}_{\text {CTE11 }}$ & 120,14 & 0,09 & 12,20 & 0,0002 & 0,0015 & 1,4515 \\
\hline
\end{tabular}

Tabla 3. Atenuación para guía de onda circular llena de aire de $1.5 \mathrm{~cm}$ de radio en el modo TEO1. Elaboración propia. 
Cuadro 1. Curva de atenuación para la guía de onda de $1.5 \mathrm{~cm}$ de radio en el modo TE01

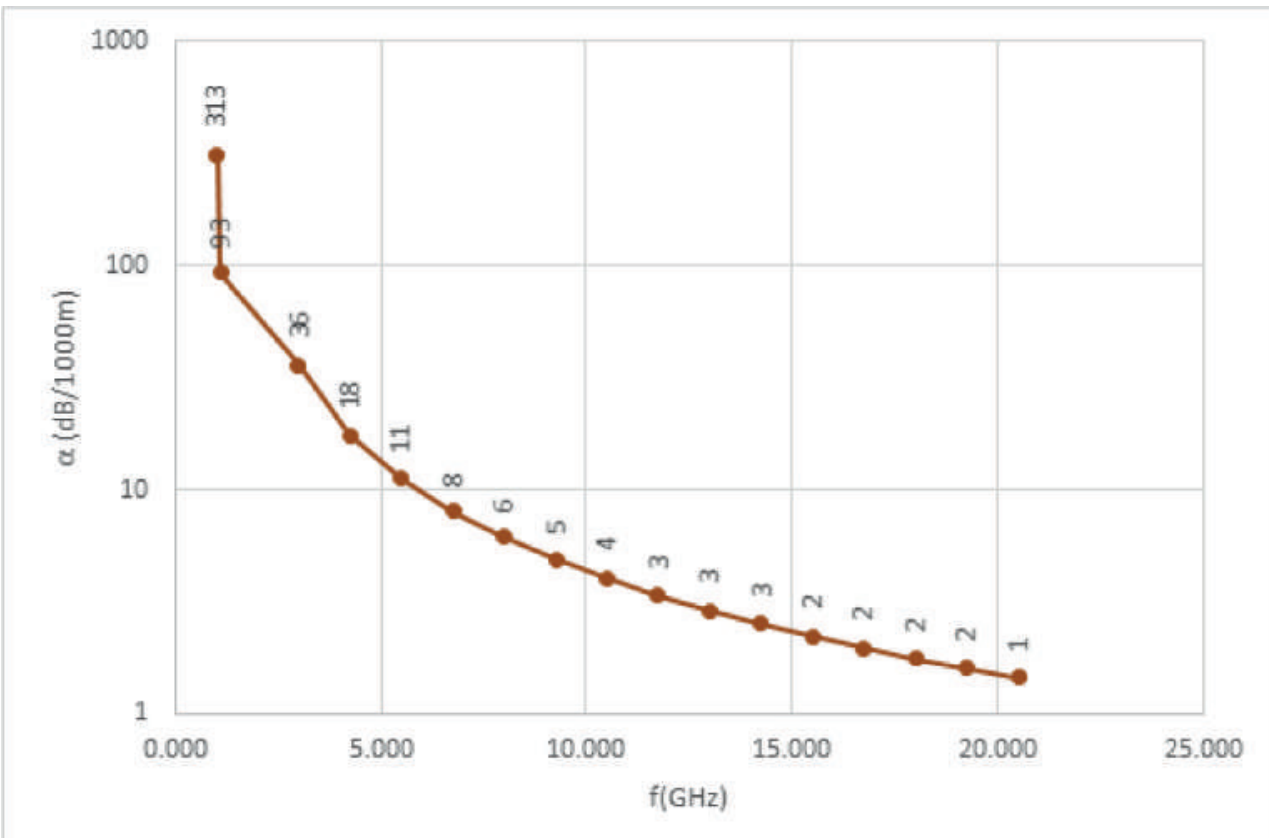

Fuente: Elaboración propia

\begin{tabular}{|c|c|c|c|c|c|c|}
\hline & $\begin{array}{c}\mathrm{f} \\
(\mathrm{GHz})\end{array}$ & $\begin{array}{c}\mathrm{R}_{\mathrm{s}} \\
\left(\mathrm{Ohmios} / \mathrm{m}^{2}\right)\end{array}$ & $\begin{array}{l}\mathrm{f}_{\text {cTE01 }} \\
(\mathrm{GHz})\end{array}$ & $(\mathrm{Np} / \mathrm{m})$ & $\begin{array}{r}\alpha \\
(\mathrm{GHz})\end{array}$ & $\left(\mathrm{Ohmios} / \mathrm{m}^{2}\right)$ \\
\hline $1,005 \mathrm{f}_{\mathrm{cTE} 02}$ & 22,44 & 0,04 & 22,33 & 0,0693 & 0,6018 & 601,8135 \\
\hline $1,010 f_{\text {СТЕ } 02}$ & 22,55 & 0,04 & 22,33 & 0,0488 & 0,4240 & 423,9634 \\
\hline $1,100 f_{\text {стЕ } 02}$ & 24,56 & 0,04 & 22,33 & 0,0145 & 0,1257 & 125,6843 \\
\hline $5,000 f_{\text {CTE } 11}$ & 29,30 & 0,05 & 22,33 & 0,0071 & 0,0621 & 62,0641 \\
\hline $6,250 \mathrm{f}_{\text {CTE} 11}$ & 36,63 & 0,05 & 22,33 & 0,0042 & 0,0363 & 36,2762 \\
\hline $7,500 \mathrm{f}_{\text {CTE11 }}$ & 43,96 & 0,06 & 22,33 & 0,0029 & 0,0254 & 25,3964 \\
\hline $8,750 \mathrm{f}_{\text {CTE11 }}$ & 51,28 & 0,06 & 22,33 & 0,0022 & 0,0193 & 19,2832 \\
\hline $10,000 \mathrm{f}_{\mathrm{CTE} 11}$ & 58,61 & 0,06 & 22,33 & 0,0018 & 0,0154 & 15,3673 \\
\hline $11,250 \mathrm{f}_{\mathrm{CTE} 11}$ & 65,93 & 0,07 & 22,33 & 0,0015 & 0,0127 & 12,6550 \\
\hline $12,500 \mathrm{f}_{\text {СТE } 11}$ & 73,26 & 0,07 & 22,33 & 0,0012 & 0,0107 & 10,6744 \\
\hline $13,750 \mathrm{f}_{\mathrm{CTE} 11}$ & 80,58 & 0,07 & 22,33 & 0,0011 & 0,0092 & 9,1713 \\
\hline $15,000 f_{\text {CTE } 11}$ & 87,91 & 0,08 & 22,33 & 0,0009 & 0,0080 & 7,9961 \\
\hline $16,250 \mathrm{f}_{\mathrm{CTE} 11}$ & 95,24 & 0,08 & 22,33 & 0,0008 & 0,0071 & 7,0556 \\
\hline $17,500 \mathrm{f}_{\mathrm{CTE} 11}$ & 102,56 & 0,08 & 22,33 & 0,0007 & 0,0063 & 6,2881 \\
\hline $18,750 \mathrm{f}_{\mathrm{CTE} 11}$ & 109,89 & 0,09 & 22,33 & 0,0007 & 0,0057 & 5,6518 \\
\hline $20,000 f_{\text {CTE } 11}$ & 117,21 & 0,09 & 22,33 & 0,0006 & 0,0051 & 5,1170 \\
\hline $21,250 \mathrm{f}_{\mathrm{CTE} 11}$ & 124,54 & 0,09 & 22,33 & 0,0005 & 0,0047 & 4,6622 \\
\hline
\end{tabular}

Tabla 4. Atenuación para guía de onda circular llena de aire, de $1.5 \mathrm{~cm}$ de radio, en el modo TE02. Elaboración propia. 
CuAdro 2. Curva de atenuación para la guía de onda de $1.5 \mathrm{~cm}$ de radio en el modo TE02

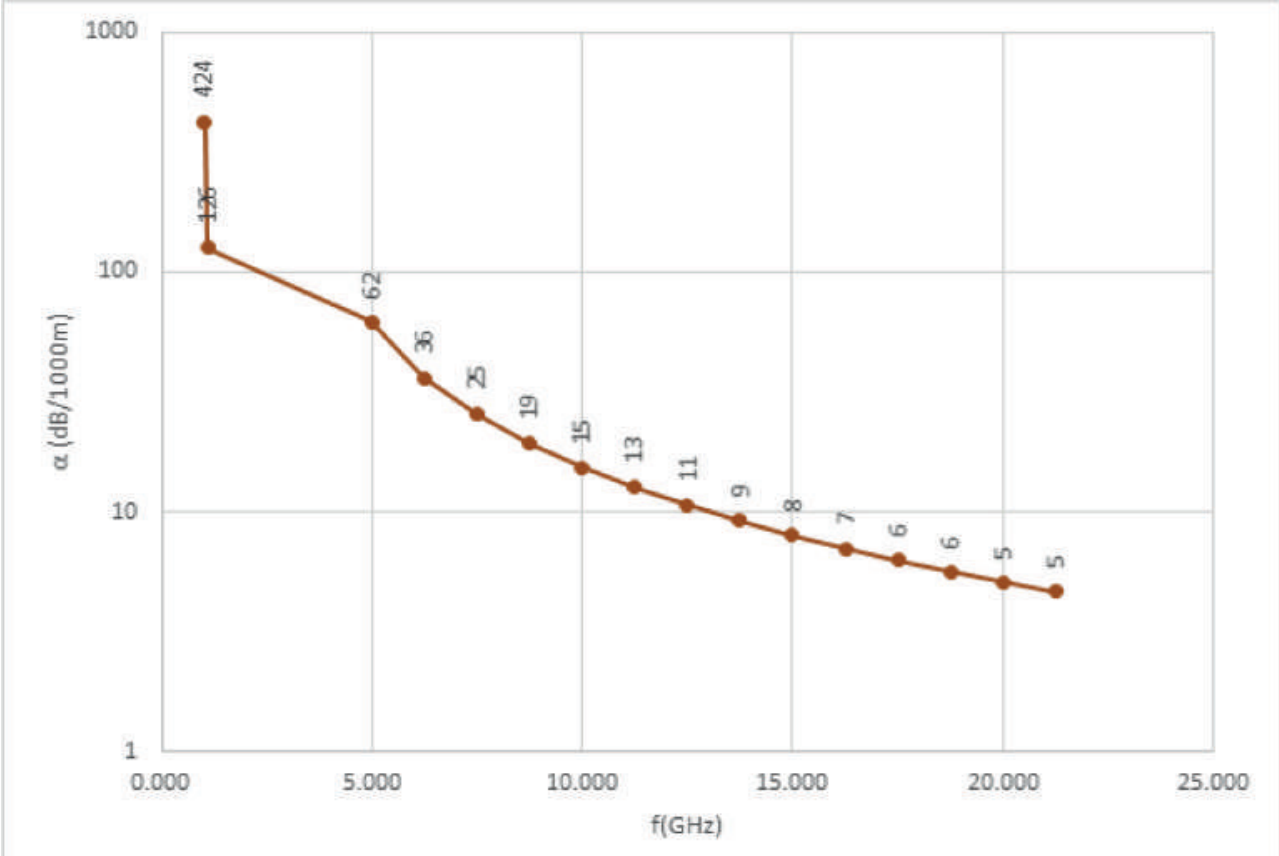

Fuente: Elaboración propia.

\begin{tabular}{|c|c|c|c|c|c|c|}
\hline & $\begin{array}{c}\mathrm{f} \\
(\mathrm{GHz})\end{array}$ & $\begin{array}{c}\mathrm{R}_{\mathrm{s}} \\
\left(\mathrm{Ohmios} / \mathrm{m}^{2}\right)\end{array}$ & $\begin{array}{l}\mathrm{f}_{\text {сTE01 }} \\
(\mathrm{GHz})\end{array}$ & $(\mathrm{Np} / \mathrm{m})$ & $\begin{array}{c}\alpha \\
(\mathrm{GHz})\end{array}$ & $\left(\mathrm{Ohmios} / \mathrm{m}^{2}\right)$ \\
\hline $1,010_{\text {cTE } 11}$ & 5,92 & 0,02 & 5,86 & 0,0357 & 0,3099 & 309,8951 \\
\hline $1,100_{\text {cTE1 } 1}$ & 6,45 & 0,02 & 5,86 & 0,0112 & 0,0970 & 96,9846 \\
\hline $1,500_{\text {cTE1 } 1}$ & 8,79 & 0,02 & 5,86 & 0,0051 & 0,0439 & 43,8756 \\
\hline $2,750_{\text {cTE } 11}$ & 16,12 & 0,03 & 5,86 & 0,0035 & 0,0303 & 30,3344 \\
\hline $4,000_{\text {cTE11 }}$ & 23,44 & 0,04 & 5,86 & 0,0035 & 0,0307 & 30,7405 \\
\hline $5,250_{\text {cTE } 11}$ & 30,77 & 0,05 & 5,86 & 0,0038 & 0,0328 & 32,8415 \\
\hline $6,500_{\text {cTE11 }}$ & 38,09 & 0,05 & 5,86 & 0,0041 & 0,0353 & 35,2988 \\
\hline $7,750_{\text {сTE } 11}$ & 45,42 & 0,06 & 5,86 & 0,0044 & 0,0378 & 37,7961 \\
\hline $9,000_{\text {cTE } 11}$ & 52,75 & 0,06 & 5,86 & 0,0046 & 0,0402 & 40,2394 \\
\hline $10,250_{\text {сTE } 11}$ & 60,07 & 0,06 & 5,86 & 0,0049 & 0,0426 & 42,6002 \\
\hline $11,500_{\text {сTE } 11}$ & 67,40 & 0,07 & 5,86 & 0,0052 & 0,0449 & 44,8724 \\
\hline $12,750_{\text {сТЕ } 11}$ & 74,72 & 0,07 & 5,86 & 0,0054 & 0,0471 & 47,0585 \\
\hline $14,000_{\text {CTE } 11}$ & 82,05 & 0,08 & 5,86 & 0,0057 & 0,0492 & 49,1635 \\
\hline $15,250_{\text {сTE } 11}$ & 89,38 & 0,08 & 5,86 & 0,0059 & 0,0512 & 51,1935 \\
\hline $16,500_{\text {сTE } 11}$ & 96,70 & 0,08 & 5,86 & 0,0061 & 0,0532 & 53,1546 \\
\hline $17,750_{\text {сTE} 11}$ & 104,03 & 0,08 & 5,86 & 0,0063 & 0,0551 & 55,0523 \\
\hline $19,000_{\text {сTE } 11}$ & 111,35 & 0,09 & 5,86 & 0,0065 & 0,0569 & 56,8917 \\
\hline $20,250_{\text {сTE } 11}$ & 118,68 & 0,09 & 5,86 & 0,0068 & 0,0587 & 58,6774 \\
\hline
\end{tabular}

Tabla 5. Atenuación para guía de onda circular llena de aire de $1.5 \mathrm{~cm}$ de radio en el modo TE11. Elaboración propia. 
Cuadro 3. Curva de atenuación para la guía de onda de $1.5 \mathrm{~cm}$ de radio en el modo TE11

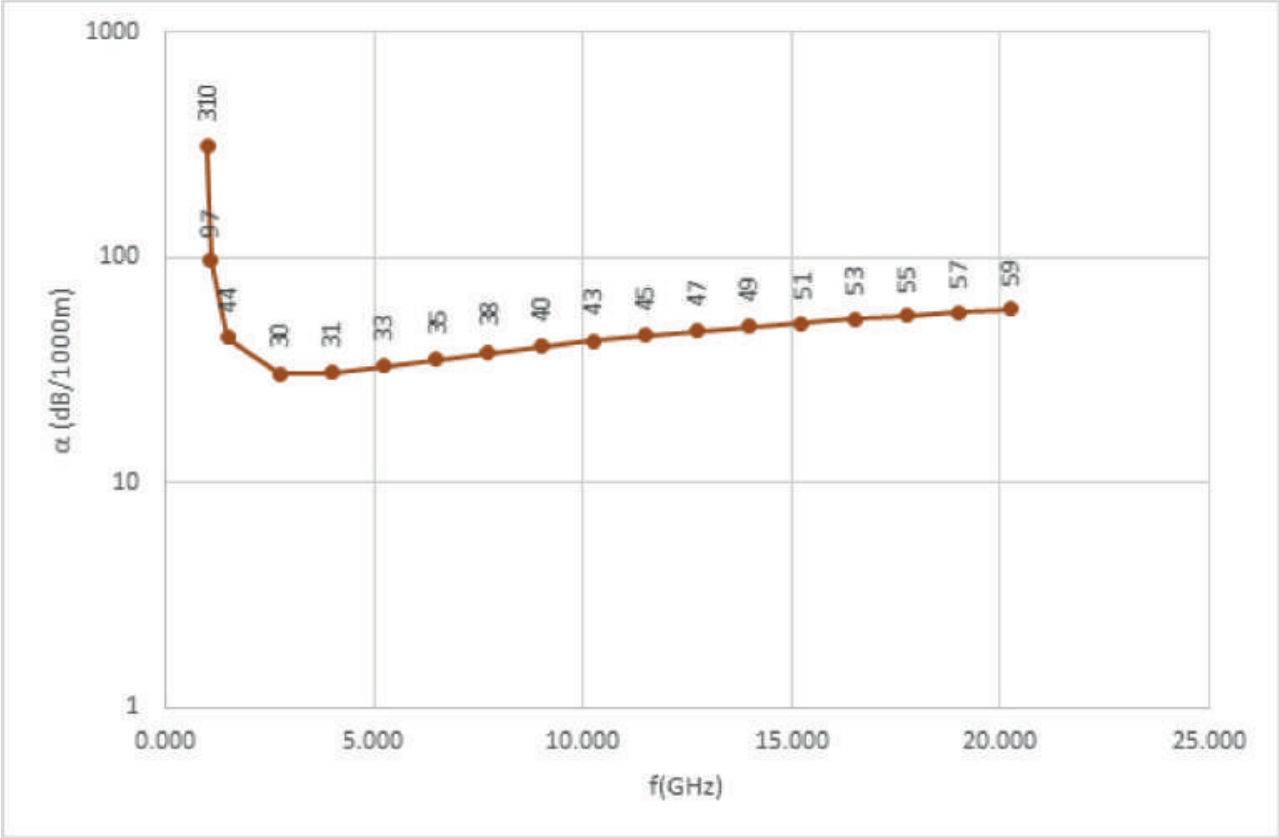

Fuente: Elaboración propia.

\begin{tabular}{|c|c|c|c|c|c|c|}
\hline & $\begin{array}{c}\mathrm{f} \\
(\mathrm{GHz})\end{array}$ & $\begin{array}{c}\mathrm{R}_{\mathrm{s}} \\
\left(\mathrm{Ohmios} / \mathrm{m}^{2}\right)\end{array}$ & $\begin{array}{l}\mathrm{f}_{\text {CTE01 }} \\
(\mathrm{GHz})\end{array}$ & $(\mathrm{Np} / \mathrm{m})$ & $\begin{array}{r}\alpha \\
(\mathrm{GHz})\end{array}$ & $\left(\mathrm{Ohmios} / \mathrm{m}^{2}\right)$ \\
\hline $1,001_{\text {сTE } 21}$ & 9,73 & 0,03 & 9,72 & 0,1797 & 1,5605 & 1560,4680 \\
\hline $1,010_{\text {сTЕ } 21}$ & 9,82 & 0,03 & 9,72 & 0,0569 & 0,4940 & 493,9596 \\
\hline $1,100_{\text {стЕ } 21}$ & 10,69 & 0,03 & 9,72 & 0,0182 & 0,1583 & 158,2572 \\
\hline $2,000_{\text {сTE11 }}$ & 11,72 & 0,03 & 9,72 & 0,0130 & 0,1127 & 112,7029 \\
\hline $3,250_{\text {сTE11 }}$ & 19,05 & 0,04 & 9,72 & 0,0076 & 0,0656 & 65,6049 \\
\hline $4,500_{\text {сTE11 }}$ & 26,37 & 0,04 & 9,72 & 0,0072 & 0,0626 & 62,6124 \\
\hline $5,750_{\text {сTE } 11}$ & 33,70 & 0,05 & 9,72 & 0,0074 & 0,0646 & 64,6326 \\
\hline $7,000_{\text {сTE11 }}$ & 41,03 & 0,05 & 9,72 & 0,0078 & 0,0680 & 68,0013 \\
\hline $8,250_{\text {сTE11 }}$ & 48,35 & 0,06 & 9,72 & 0,0083 & 0,0718 & 71,7889 \\
\hline $9,500_{\text {сTE11 }}$ & 55,68 & 0,06 & 9,72 & 0,0087 & 0,0757 & 75,6771 \\
\hline $10,750_{\text {cTE } 11}$ & 63,00 & 0,07 & 9,72 & 0,0092 & 0,0795 & 79,5404 \\
\hline $12,000_{\text {сTE } 11}$ & 70,33 & 0,07 & 9,72 & 0,0096 & 0,0833 & 83,3270 \\
\hline $13,250_{\text {cTE11 }}$ & 77,65 & 0,07 & 9,72 & 0,0100 & 0,0870 & 87,0165 \\
\hline $14,500_{\text {сTE11 }}$ & 84,98 & 0,08 & 9,72 & 0,0104 & 0,0906 & 90,6025 \\
\hline $15,750_{\text {cTE11 }}$ & 92,31 & 0,08 & 9,72 & 0,0108 & 0,0941 & 94,0854 \\
\hline $17,000_{\text {сTE11 }}$ & 99,63 & 0,08 & 9,72 & 0,0112 & 0,0975 & 97,4687 \\
\hline $18,250_{\text {cTE } 11}$ & 106,96 & 0,09 & 9,72 & 0,0116 & 0,1008 & 100,7571 \\
\hline $19,500_{\text {cTE11 }}$ & 114,28 & 0,09 & 9,72 & 0,0120 & 0,1040 & 103,9562 \\
\hline $20,750_{\text {сTЕ } 11}$ & 121,61 & 0,09 & 9,72 & 0,0123 & 0,1071 & 107,0711 \\
\hline
\end{tabular}

Tabla 6. Atenuación para guía de onda circular llena de aire de $1.5 \mathrm{~cm}$ de radio en el modo TE21. Elaboración propia. 
CuAdro 4. Curva de atenuación para la guía de onda de $1.5 \mathrm{~cm}$ de radio en el modo TE21

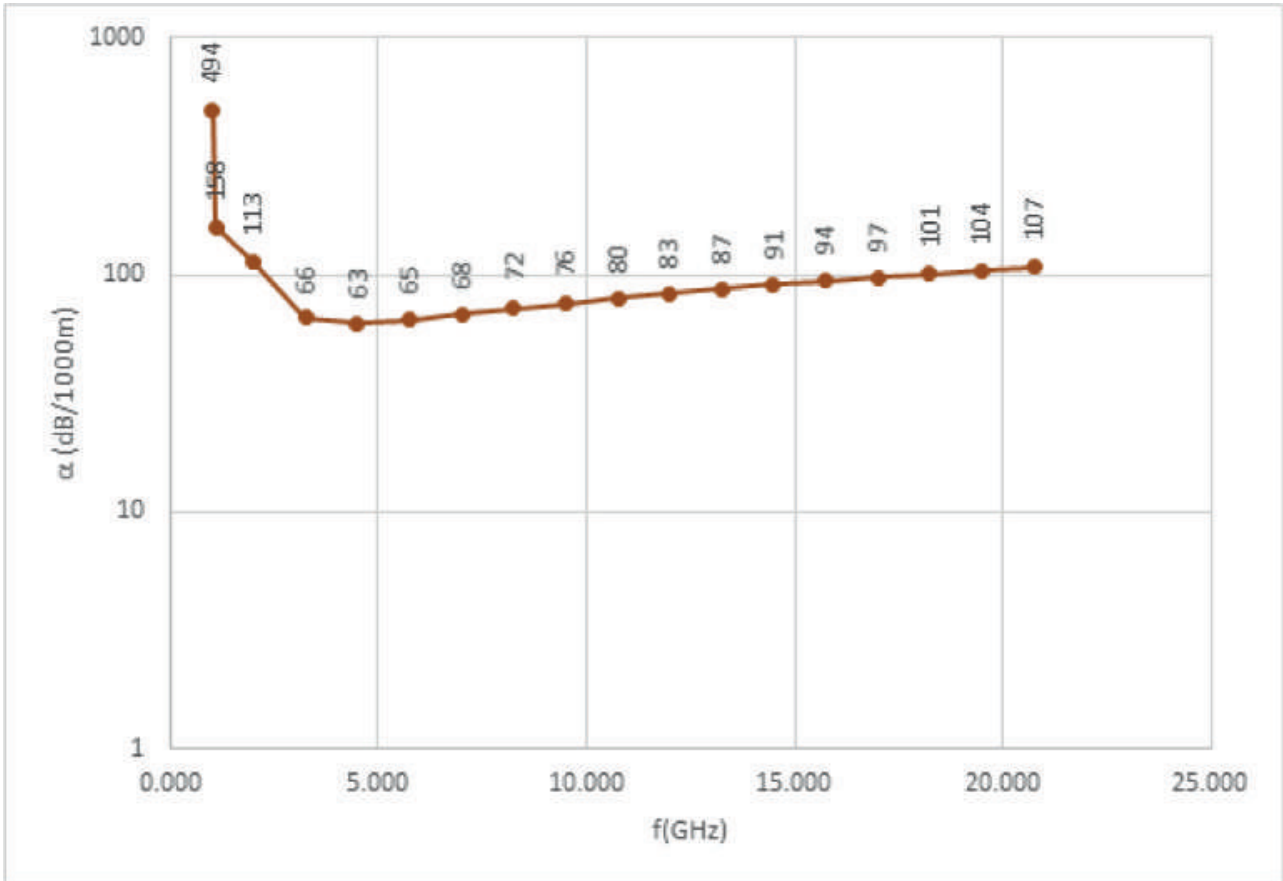

Fuente: Elaboración propia.

\begin{tabular}{|c|c|c|c|c|c|c|}
\hline & $\begin{array}{c}\mathrm{f} \\
(\mathrm{GHz})\end{array}$ & $\begin{array}{c}\mathrm{R}_{\mathrm{s}} \\
\left(\mathrm{Ohmios} / \mathrm{m}^{2}\right)\end{array}$ & $\begin{array}{r}\mathrm{f}_{\text {CTE01 }} \\
(\mathrm{GHz})\end{array}$ & $(\mathrm{Np} / \mathrm{m})$ & $(\mathrm{GHz})^{\alpha}$ & $\left(\mathrm{Ohmios} / \mathrm{m}^{2}\right)$ \\
\hline $1,001_{\text {стм01 }}$ & 7,66 & 0,02 & 7,66 & 0,0912 & 0,7918 & 791,8279 \\
\hline $1,010_{\text {стм01 }}$ & 7,73 & 0,02 & 7,66 & 0,0292 & 0,2532 & 253,2137 \\
\hline $1,100_{\text {стМ } 01}$ & 8,42 & 0,02 & 7,66 & 0,0103 & 0,0890 & 89,0395 \\
\hline $2,000_{\text {сTE11 }}$ & 11,72 & 0,03 & 7,66 & 0,0067 & 0,0578 & 57,7908 \\
\hline $3,250_{\text {cTE11 }}$ & 19,05 & 0,04 & 7,66 & 0,0070 & 0,0609 & 60,9257 \\
\hline $4,500_{\text {сTE11 }}$ & 26,37 & 0,04 & 7,66 & 0,0079 & 0,0686 & 68,5998 \\
\hline $5,750_{\text {cTE1 } 1}$ & 33,70 & 0,05 & 7,66 & 0,0088 & 0,0762 & 76,1980 \\
\hline $7,000_{\text {сTE1 } 1}$ & 41,03 & 0,05 & 7,66 & 0,0096 & 0,0833 & 83,3392 \\
\hline $8,250_{\text {сTE11 }}$ & 48,35 & 0,06 & 7,66 & 0,0104 & 0,0900 & 90,0212 \\
\hline $9,500_{\text {сTE11 }}$ & 55,68 & 0,06 & 7,66 & 0,0111 & 0,0963 & 96,2966 \\
\hline $10,750_{\text {сTE1 } 1}$ & 63,00 & 0,07 & 7,66 & 0,0118 & 0,1022 & 102,2208 \\
\hline $12,000_{\text {сTE } 11}$ & 70,33 & 0,07 & 7,66 & 0,0124 & 0,1078 & 107,8410 \\
\hline $13,250_{\text {cTE } 11}$ & 77,65 & 0,07 & 7,66 & 0,0130 & 0,1132 & 113,1967 \\
\hline $14,500_{\text {сTE11 }}$ & 84,98 & 0,08 & 7,66 & 0,0136 & 0,1183 & 118,3201 \\
\hline $15,750_{\text {cTE } 11}$ & 92,31 & 0,08 & 7,66 & 0,0142 & 0,1232 & 123,2378 \\
\hline $17,000_{\text {сTE1 } 1}$ & 99,63 & 0,08 & 7,66 & 0,0147 & 0,1280 & 127,9721 \\
\hline $18,250_{\text {сTE11 }}$ & 106,96 & 0,09 & 7,66 & 0,0153 & 0,1325 & 132,5415 \\
\hline $19,500_{\text {сTE1 } 1}$ & 114,28 & 0,09 & 7,66 & 0,0158 & 0,1370 & 136,9616 \\
\hline $20,750_{\text {сTE } 11}$ & 121,61 & 0,09 & 7,66 & 0,0163 & 0,1412 & 141,2461 \\
\hline
\end{tabular}

Tabla 7. Atenuación para guia de onda circular llena de aire de $1.5 \mathrm{~cm}$ de radio en el modo TM01. Elaboración propia. 
Cuadro 5. Curva de atenuación para la guía de onda de $1.5 \mathrm{~cm}$ de radio en el modo TM01

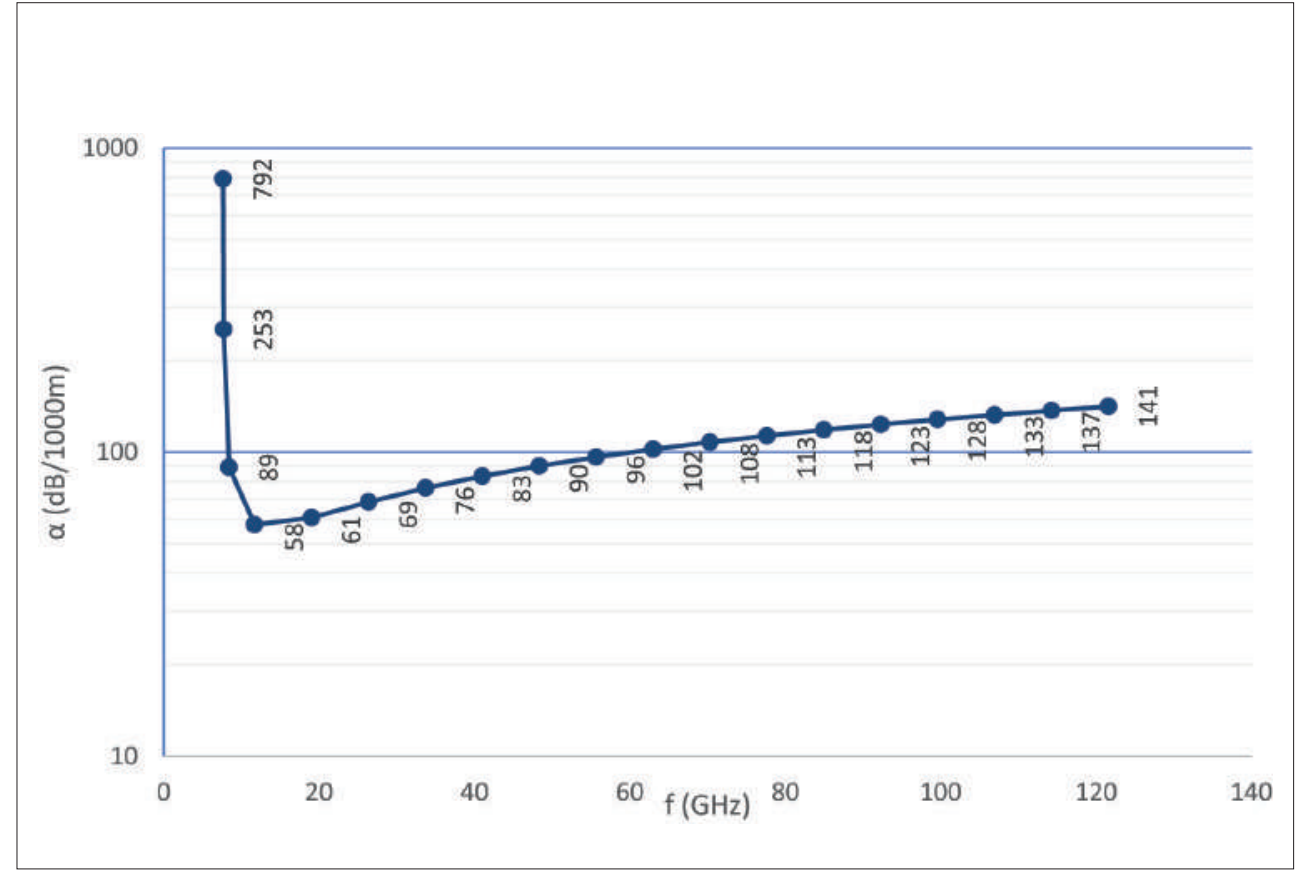

Fuente: Elaboración propia.

\begin{tabular}{|c|c|c|c|c|c|c|}
\hline & $\begin{array}{c}\mathrm{f} \\
(\mathrm{GHz})\end{array}$ & $\begin{array}{c}\mathrm{R}_{\mathrm{s}} \\
\left(\text { Ohmios } / \mathrm{m}^{2}\right)\end{array}$ & $\begin{array}{l}\mathrm{f}_{\text {CTE01 }} \\
(\mathrm{GHz})\end{array}$ & $(\mathrm{Np} / \mathrm{m})$ & $(\mathrm{GHz})^{\alpha}$ & $\left(\mathrm{Ohmios} / \mathrm{m}^{2}\right)$ \\
\hline $1,001_{\text {сTM } 11}$ & 12,21 & 0,03 & 12,20 & 0,1151 & 0,9995 & 999,5021 \\
\hline $1,010_{\text {сTM } 11}$ & 12,32 & 0,03 & 12,20 & 0,0368 & 0,3196 & 319,6246 \\
\hline $1,100_{\text {стM } 11}$ & 13,42 & 0,03 & 12,20 & 0,0129 & 0,1124 & 112,3920 \\
\hline $3,250_{\text {cTE1 } 1}$ & 19,05 & 0,04 & 12,20 & 0,0084 & 0,0726 & 72,6339 \\
\hline $4,500_{\text {cTE } 11}$ & 26,37 & 0,04 & 12,20 & 0,0085 & 0,0740 & 74,0403 \\
\hline $5,750_{\text {cTE1 } 1}$ & 33,70 & 0,05 & 12,20 & 0,0092 & 0,0796 & 79,6029 \\
\hline $7,000_{\text {cTE11 }}$ & 41,03 & 0,05 & 12,20 & 0,0099 & 0,0858 & 85,7531 \\
\hline $8,250_{\text {cTE } 11}$ & 48,35 & 0,06 & 12,20 & 0,0106 & 0,0919 & 91,8564 \\
\hline $9,500_{\text {cTE } 11}$ & 55,68 & 0,06 & 12,20 & 0,0113 & 0,0978 & 97,7567 \\
\hline $10,750_{\text {сTE1 } 1}$ & 63,00 & 0,07 & 12,20 & 0,0119 & 0,1034 & 103,4199 \\
\hline $12,000_{\text {сTE } 11}$ & 70,33 & 0,07 & 12,20 & 0,0125 & 0,1088 & 108,8497 \\
\hline $13,250_{\text {сTE } 11}$ & 77,65 & 0,07 & 12,20 & 0,0131 & 0,1141 & 114,0611 \\
\hline $14,500_{\text {сTE} 11}$ & 84,98 & 0,08 & 12,20 & 0,0137 & 0,1191 & 119,0719 \\
\hline $15,750_{\text {стЕ } 11}$ & 92,31 & 0,08 & 12,20 & 0,0143 & 0,1239 & 123,8997 \\
\hline $17,000_{\text {сTE11 }}$ & 99,63 & 0,08 & 12,20 & 0,0148 & 0,1286 & 128,5608 \\
\hline $18,250_{\text {сTE } 11}$ & 106,96 & 0,09 & 12,20 & 0,0153 & 0,1331 & 133,0696 \\
\hline $19,500_{\text {сTE } 11}$ & 114,28 & 0,09 & 12,20 & 0,0158 & 0,1374 & 137,4390 \\
\hline $20,750_{\text {сTE } 11}$ & 121,61 & 0,09 & 12,20 & 0,0163 & 0,1417 & 141,6804 \\
\hline
\end{tabular}

Tabla 8. Atenuación para guía de onda circular llena de aire de $1.5 \mathrm{~cm}$ de radio en el modo TM11. Elaboración propia. 


\begin{tabular}{|c|c|c|c|c|c|c|}
\hline $\mathrm{f} / \mathrm{f}_{\mathrm{cTE} 11}$ & $\begin{array}{c}\alpha_{\mathrm{TE} 01} \\
(\mathrm{~dB} / 1000 \mathrm{~m})\end{array}$ & $\begin{array}{c}\alpha_{\text {TE02 }} \\
(\mathrm{dB} / 1000 \mathrm{~m})\end{array}$ & $\begin{array}{c}\alpha_{\mathrm{TE} 11} \\
(\mathrm{~dB} / 1000 \mathrm{~m})\end{array}$ & $\begin{array}{c}\alpha_{\text {TE21 }} \\
(\mathrm{dB} / 1000 \mathrm{~m})\end{array}$ & $\begin{array}{c}\alpha_{\mathrm{TM} 01} \\
(\mathrm{~dB} / 1000 \mathrm{~m})\end{array}$ & $\begin{array}{c}\alpha_{\text {TM11 }} \\
(\mathrm{dB} / 1000 \mathrm{~m})\end{array}$ \\
\hline 2.00 & & & 33.7777 & 112.7029 & 57.7908 & \\
\hline 2.50 & & & 30.8794 & 77.8921 & 57.3850 & 88.3070 \\
\hline 3.00 & 35.8132 & & 30.1039 & 67.9613 & 59.5396 & 74.4187 \\
\hline 3.50 & 25.4592 & & 30.2089 & 64.1278 & 62.4030 & 72.0073 \\
\hline 4.00 & 19.6185 & 184.5786 & 30.7405 & 62.7595 & 65.4816 & 72.4738 \\
\hline 4.50 & 15.8361 & 88.4708 & 31.4963 & 62.6124 & 68.5998 & 74.0403 \\
\hline 5.00 & 13.1846 & 62.0641 & 32.3747 & 63.1411 & 71.6867 & 76.1031 \\
\hline 5.50 & 11.2259 & 48.3025 & 33.3204 & 64.0694 & 74.7123 & 78.4046 \\
\hline 6.00 & 9.7234 & 39.5756 & 34.3015 & 65.2439 & 77.6646 & 80.8195 \\
\hline 6.50 & 8.5374 & 33.4650 & 35.2988 & 66.5734 & 80.5401 & 83.2813 \\
\hline 7.00 & 7.5798 & 28.9196 & 36.3006 & 68.0013 & 83.3392 & 85.7531 \\
\hline 7.50 & 6.7923 & 25.3964 & 37.2994 & 69.4907 & 86.0645 & 88.2134 \\
\hline 8.00 & 6.1347 & 22.5823 & 38.2906 & 71.0172 & 88.7191 & 90.6497 \\
\hline 8.50 & 5.5783 & 20.2823 & 39.2712 & 72.5642 & 91.3069 & 93.0547 \\
\hline 9.00 & 5.1024 & 18.3680 & 40.2394 & 74.1202 & 93.8316 & 95.4244 \\
\hline 9.50 & 4.6914 & 16.7507 & 41.1943 & 75.6771 & 96.2966 & 97.7567 \\
\hline 10.00 & 4.3333 & 15.3673 & 42.1351 & 77.2293 & 98.7056 & 100.0507 \\
\hline 10.50 & 4.0191 & 14.1714 & 43.0617 & 78.7728 & 101.0616 & 102.3063 \\
\hline 11.00 & 3.7414 & 13.1280 & 43.9741 & 80.3048 & 103.3677 & 104.5242 \\
\hline 11.50 & 3.4946 & 12.2106 & 44.8724 & 81.8233 & 105.6267 & 106.7050 \\
\hline 12.00 & 3.2739 & 11.3983 & 45.7569 & 83.3270 & 107.8410 & 108.8497 \\
\hline 12.50 & 3.0757 & 10.6744 & 46.6279 & 84.8150 & 110.0131 & 110.9595 \\
\hline 13.00 & 2.8969 & 10.0259 & 47.4858 & 86.2868 & 112.1451 & 113.0354 \\
\hline 13.50 & 2.7348 & 9.4419 & 48.3308 & 87.7420 & 114.2390 & 115.0787 \\
\hline 14.00 & 2.5874 & 8.9136 & 49.1635 & 89.1806 & 116.2968 & 117.0905 \\
\hline 14.50 & 2.4529 & 8.4337 & 49.9842 & 90.6025 & 118.3201 & 119.0719 \\
\hline 15.00 & 2.3297 & 7.9961 & 50.7932 & 92.0079 & 120.3105 & 121.0240 \\
\hline 15.50 & 2.2165 & 7.5958 & 51.5911 & 93.3969 & 122.2696 & 122.9480 \\
\hline 16.00 & 2.1122 & 7.2282 & 52.3781 & 94.7699 & 124.1987 & 124.8448 \\
\hline 16.50 & 2.0159 & 6.8898 & 53.1546 & 96.1270 & 126.0992 & 126.7154 \\
\hline 17.00 & 1.9267 & 6.5774 & 53.9210 & 97.4687 & 127.9721 & 128.5608 \\
\hline 17.50 & 1.8439 & 6.2881 & 54.6776 & 98.7951 & 129.8187 & 130.3819 \\
\hline 18.00 & 1.7669 & 6.0198 & 55.4247 & 100.1068 & 131.6400 & 132.1794 \\
\hline 18.50 & 1.6952 & 5.7702 & 56.1627 & 101.4039 & 133.4370 & 133.9543 \\
\hline 19.00 & 1.6282 & 5.5375 & 56.8917 & 102.6870 & 135.2106 & 135.7072 \\
\hline 19.50 & 1.5655 & 5.3203 & 57.6122 & 103.9562 & 136.9616 & 137.4390 \\
\hline 20.00 & 1.5067 & 5.1170 & 58.3243 & 105.2120 & 138.6910 & 139.1503 \\
\hline
\end{tabular}

Tabla 9. Comparación entre las atenuaciones de diversos modos de transmisión TE y TM para guía de onda circular llena de aire de $3 \mathrm{~cm}$ de radio. Elaboración propia. 
Cuadro 6. Curva de atenuación para la guía de onda de $1.5 \mathrm{~cm}$ de radio en el modo TM11

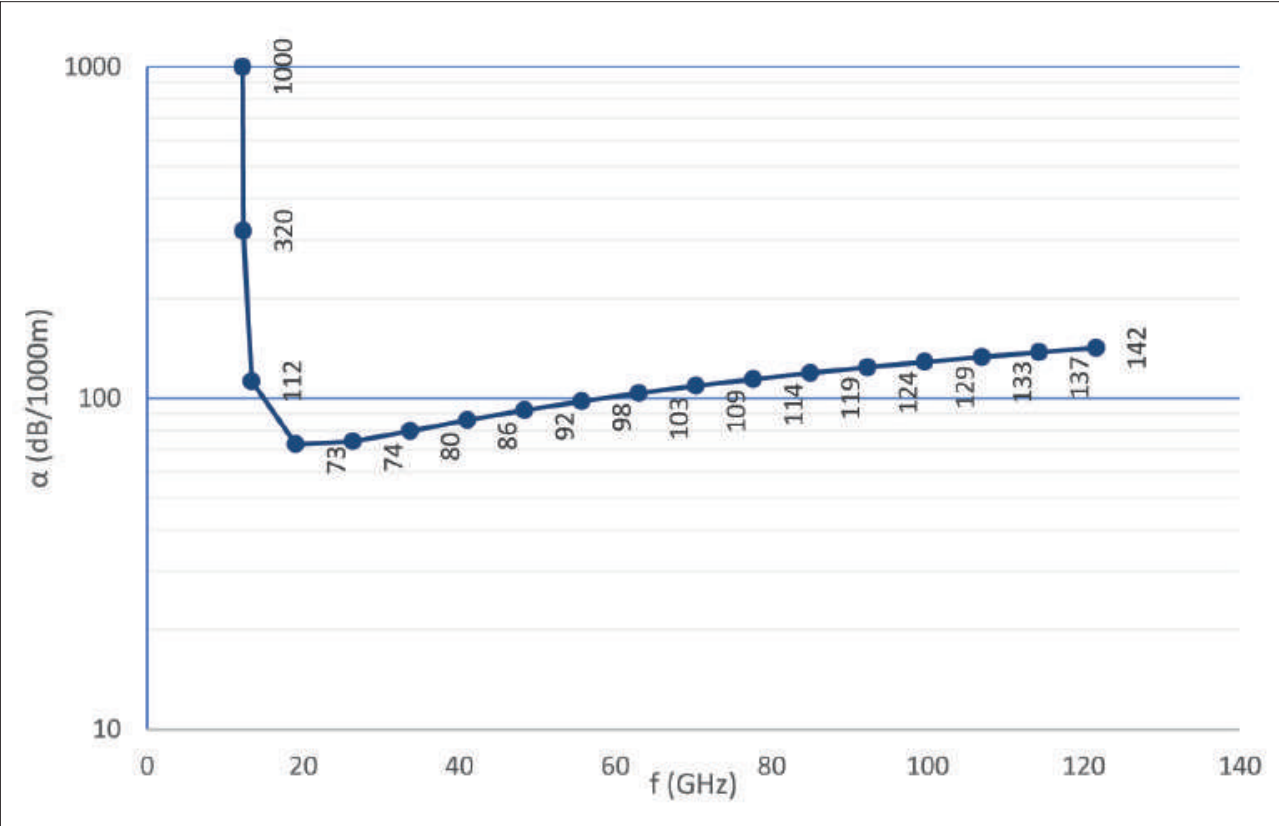

Fuente: Elaboración propia.

CuAdro 7. Curva de atenuación para la guía de onda de $1.5 \mathrm{~cm}$ de radio

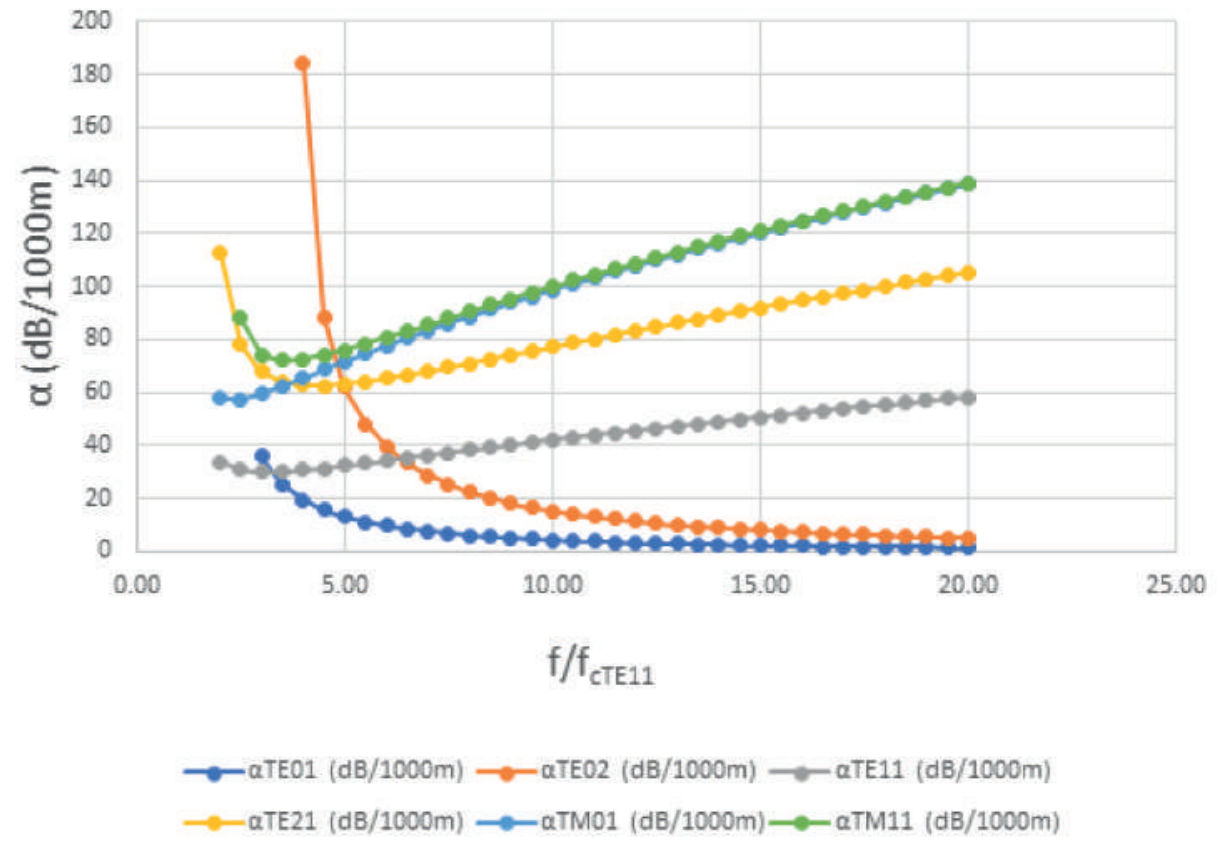

Fuente: Elaboración propia. 


\begin{tabular}{|c|c|c|c|c|c|c|}
\hline & $\begin{array}{c}\mathrm{f} \\
(\mathrm{GHz})\end{array}$ & $\begin{array}{c}\mathrm{R}_{\mathrm{s}} \\
\left(\mathrm{Ohmios} / \mathrm{m}^{2}\right)\end{array}$ & $\begin{array}{c}\mathrm{f}_{\mathrm{cTE} 01} \\
(\mathrm{GHz})\end{array}$ & $(\mathrm{Np} / \mathrm{m})$ & $\begin{array}{r}\alpha \\
(\mathrm{GHz})\end{array}$ & $\left(\mathrm{Ohmios} / \mathrm{m}^{2}\right)$ \\
\hline $1,005_{\mathrm{cTE} 01}$ & 6,13 & 0,02 & 6,10 & 0,0181 & 0,1572 & 157,2483 \\
\hline $1,010_{\text {стЕ } 01}$ & 6,16 & 0,02 & 6,10 & 0,0128 & 0,1108 & 110,7777 \\
\hline $1,100_{\text {сTЕ } 01}$ & 6,71 & 0,02 & 6,10 & 0,0038 & 0,0328 & 32,8402 \\
\hline $3,000_{\text {сTE11 }}$ & 8,79 & 0,02 & 6,10 & 0,0015 & 0,0127 & 12,6619 \\
\hline $4,250_{\text {cTE11 }}$ & 12,45 & 0,03 & 6,10 & 0,0007 & 0,0062 & 6,2032 \\
\hline $5,500_{\text {cTE11 }}$ & 16,12 & 0,03 & 6,10 & 0,0005 & 0,0040 & 3,9689 \\
\hline $6,750_{\text {cTE } 11}$ & 19,78 & 0,04 & 6,10 & 0,0003 & 0,0028 & 2,8405 \\
\hline $8,000_{\text {сTE } 11}$ & 23,44 & 0,04 & 6,10 & 0,0002 & 0,0022 & 2,1689 \\
\hline 9,250 сTE11 & 27,11 & 0,04 & 6,10 & 0,0002 & 0,0017 & 1,7287 \\
\hline $10,500_{\text {сTE } 11}$ & 30,77 & 0,05 & 6,10 & 0,0002 & 0,0014 & 1,4210 \\
\hline 11,750 сTE11 & 34,43 & 0,05 & 6,10 & 0,0001 & 0,0012 & 1,1954 \\
\hline $13,000_{\text {сTE } 11}$ & 38,09 & 0,05 & 6,10 & 0,0001 & 0,0010 & 1,0242 \\
\hline $14,250_{\text {сTE } 11}$ & 41,76 & 0,05 & 6,10 & 0,0001 & 0,0009 & 0,8905 \\
\hline $15,500_{\text {сTE } 11}$ & 45,42 & 0,06 & 6,10 & 0,0001 & 0,0008 & 0,7836 \\
\hline $16,750_{\text {сTE } 11}$ & 49,08 & 0,06 & 6,10 & 0,0001 & 0,0007 & 0,6967 \\
\hline $18,000_{\text {сTE11 }}$ & 52,75 & 0,06 & 6,10 & 0,0001 & 0,0006 & 0,6247 \\
\hline $19,250_{\text {сTE } 11}$ & 56,41 & 0,06 & 6,10 & 0,0001 & 0,0006 & 0,5644 \\
\hline $20,500_{\text {сTE } 11}$ & 60,07 & 0,06 & 6,10 & 0,0001 & 0,0005 & 0,5132 \\
\hline
\end{tabular}

Tabla 10. Atenuación para guía de onda circular llena de aire de $3 \mathrm{~cm}$ de radio en el modo TEO1. Elaboración propia.

CuAdro 8. Curva de atenuación para la guía de onda de $3 \mathrm{~cm}$ de radio en el modo TE01

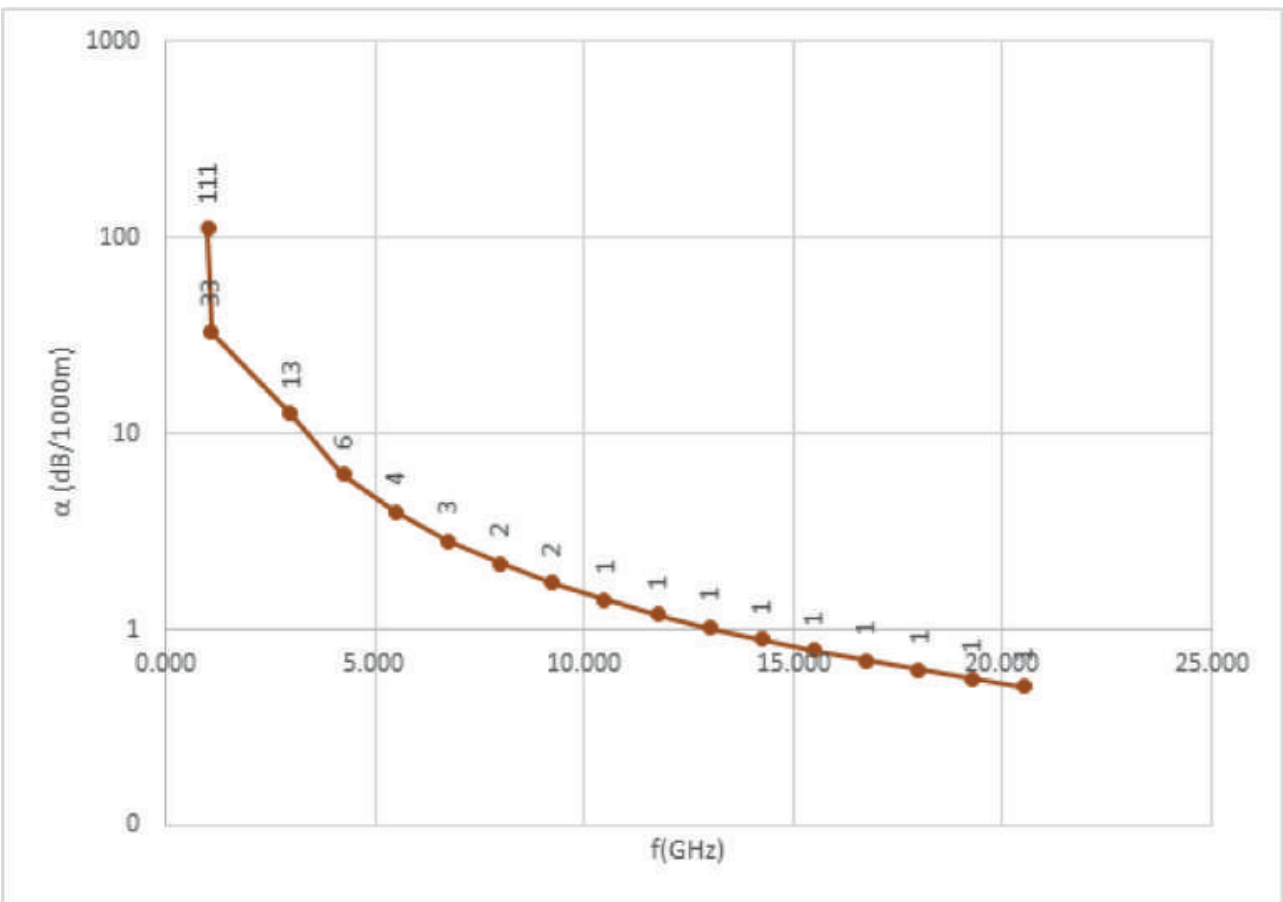

Fuente: Elaboración propia. 


\begin{tabular}{|c|c|c|c|c|c|c|}
\hline & $\begin{array}{c}\mathrm{f} \\
(\mathrm{GHz})\end{array}$ & $\begin{array}{c}\mathrm{R}_{\mathrm{s}} \\
\left(\mathrm{Ohmios} / \mathrm{m}^{2}\right)\end{array}$ & $\begin{array}{l}\mathrm{f}_{\mathrm{cTE} 01} \\
(\mathrm{GHz})\end{array}$ & $(\mathrm{Np} / \mathrm{m})$ & $\begin{array}{r}\alpha \\
(\mathrm{GHz})\end{array}$ & $\left(\mathrm{Ohmios} / \mathrm{m}^{2}\right)$ \\
\hline $1,005_{\text {стЕ } 02}$ & 11,22 & 0,03 & 11,17 & 0,0245 & 0,2128 & 212,7732 \\
\hline $1,010_{\text {стЕ} 02}$ & 11,28 & 0,03 & 11,17 & 0,0173 & 0,1499 & 149,8937 \\
\hline $1,100_{\text {стЕ } 02}$ & 12,28 & 0,03 & 11,17 & 0,0051 & 0,0444 & 44,4361 \\
\hline $5,000_{\text {сTE11 }}$ & 14,65 & 0,03 & 11,17 & 0,0025 & 0,0219 & 21,9430 \\
\hline $6,250_{\text {сTE11 }}$ & 18,31 & 0,04 & 11,17 & 0,0015 & 0,0128 & 12,8256 \\
\hline $7,500_{\text {сTE11 }}$ & 21,98 & 0,04 & 11,17 & 0,0010 & 0,0090 & 8,9790 \\
\hline $8,750_{\text {сTE11 }}$ & 25,64 & 0,04 & 11,17 & 0,0008 & 0,0068 & 6,8176 \\
\hline $10,000_{\text {сTE11 }}$ & 29,30 & 0,05 & 11,17 & 0,0006 & 0,0054 & 5,4332 \\
\hline $11,250_{\text {cTE11 }}$ & 32,97 & 0,05 & 11,17 & 0,0005 & 0,0045 & 4,4742 \\
\hline $12,500_{\text {cTE11 }}$ & 36,63 & 0,05 & 11,17 & 0,0004 & 0,0038 & 3,7740 \\
\hline $13,750_{\text {cTE11 }}$ & 40,29 & 0,05 & 11,17 & 0,0004 & 0,0032 & 3,2425 \\
\hline $15,000_{\text {сTE11 }}$ & 43,96 & 0,06 & 11,17 & 0,0003 & 0,0028 & 2,8271 \\
\hline $16,250_{\text {сTE11 }}$ & 47,62 & 0,06 & 11,17 & 0,0003 & 0,0025 & 2,4945 \\
\hline $17,500_{\text {сTE11 }}$ & 51,28 & 0,06 & 11,17 & 0,0003 & 0,0022 & 2,2232 \\
\hline $18,750_{\text {cTE11 }}$ & 54,94 & 0,06 & 11,17 & 0,0002 & 0,0020 & 1,9982 \\
\hline $20,000_{\text {cTE11 }}$ & 58,61 & 0,06 & 11,17 & 0,0002 & 0,0018 & 1,8091 \\
\hline $21,250_{\text {cTE } 11}$ & 62,27 & 0,07 & 11,17 & 0,0002 & 0,0016 & 1,6483 \\
\hline
\end{tabular}

Tabla 11. Atenuación para guía de onda circular llena de aire de $3 \mathrm{~cm}$ de radio en el modo TE02. Elaboración propia.

Cuadro 9. Curva de atenuación para la guía de onda de $3 \mathrm{~cm}$ de radio en el modo TE02

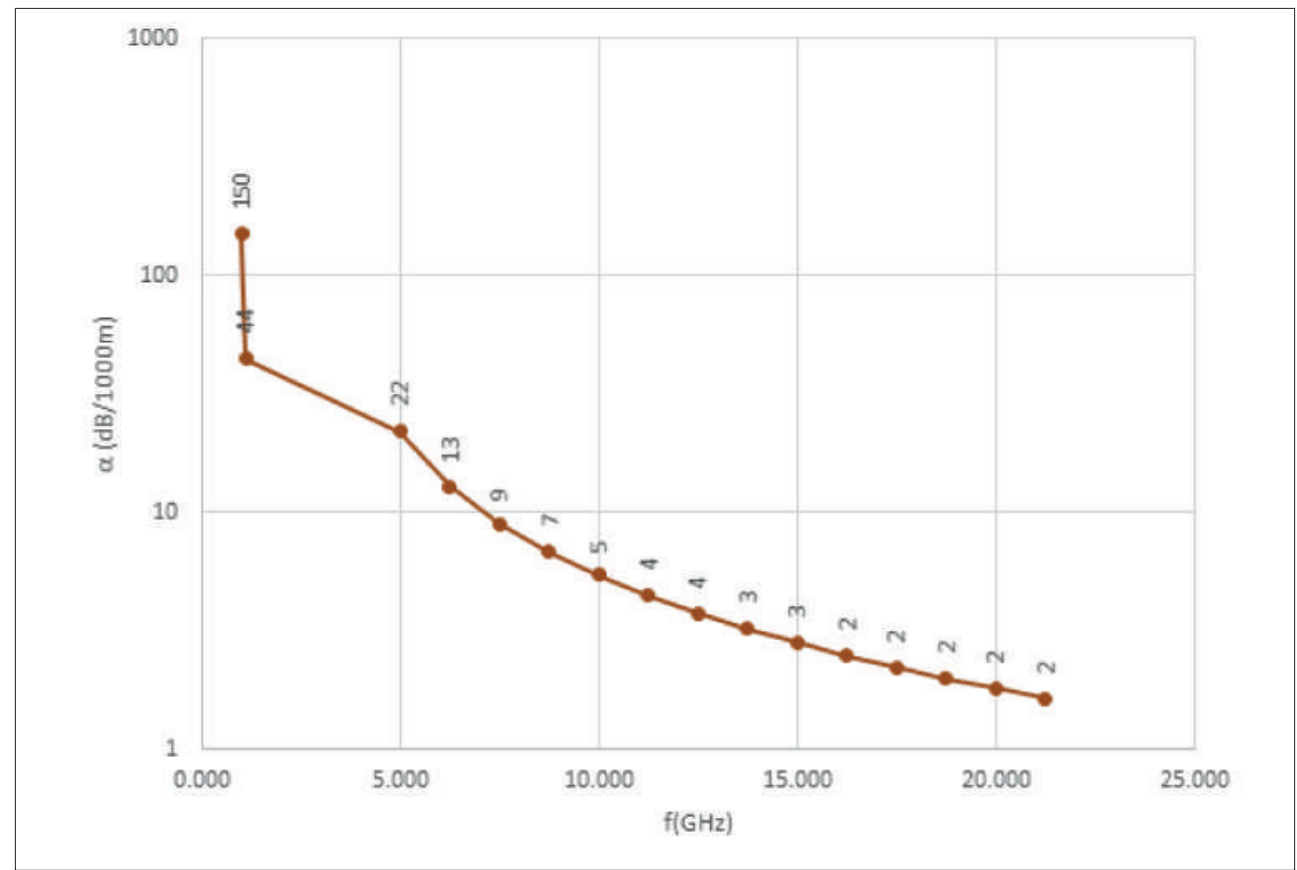

Fuente: Elaboración propia. 


\begin{tabular}{lcccccr}
\hline & $\begin{array}{c}\mathrm{f} \\
(\mathrm{GHz})\end{array}$ & $\begin{array}{c}\mathrm{R}_{\mathrm{s}} \\
\left(\mathrm{Ohmios} / \mathrm{m}^{2}\right)\end{array}$ & $\begin{array}{c}\mathrm{f}_{\mathrm{cTE} 01} \\
(\mathrm{GHz})\end{array}$ & $\begin{array}{c}\alpha \\
(\mathrm{Np} / \mathrm{m})\end{array}$ & $\begin{array}{c}\mathrm{GHz}) \\
\left(\mathrm{Ohmios} / \mathrm{m}^{2}\right)\end{array}$ \\
\hline $1,010_{\text {cTE11 }}$ & 2,96 & 0,01 & 2,93 & 0,0126 & 0,1096 & 109,5645 \\
$1,100_{\text {cTE11 }}$ & 3,22 & 0,01 & 2,93 & 0,0039 & 0,0343 & 34,2892 \\
$1,500_{\text {cTE11 }}$ & 4,40 & 0,02 & 2,93 & 0,0018 & 0,0155 & 15,5124 \\
$2,750_{\text {cTE11 }}$ & 8,06 & 0,02 & 2,93 & 0,0012 & 0,0107 & 10,7248 \\
$4,000_{\text {cTE11 }}$ & 11,72 & 0,03 & 2,93 & 0,0013 & 0,0109 & 10,8684 \\
$5,250_{\text {cTE11 }}$ & 15,38 & 0,03 & 2,93 & 0,0013 & 0,0116 & 11,6112 \\
$6,500_{\text {cTE11 }}$ & 19,05 & 0,04 & 2,93 & 0,0014 & 0,0125 & 12,4800 \\
$7,750_{\text {cTE11 }}$ & 22,71 & 0,04 & 2,93 & 0,0015 & 0,0134 & 13,3629 \\
$9,000_{\text {cTE11 }}$ & 26,37 & 0,04 & 2,93 & 0,0016 & 0,0142 & 14,2268 \\
$10,250_{\text {cTE11 }}$ & 30,04 & 0,05 & 2,93 & 0,0017 & 0,0151 & 15,0614 \\
$11,500_{\text {cTE11 }}$ & 33,70 & 0,05 & 2,93 & 0,0018 & 0,0159 & 15,8648 \\
$12,750_{\text {cTE11 }}$ & 37,36 & 0,05 & 2,93 & 0,0019 & 0,0166 & 16,6377 \\
$14,000_{\text {cTE11 }}$ & 41,03 & 0,05 & 2,93 & 0,0020 & 0,0174 & 17,3819 \\
$15,250_{\text {cTE11 }}$ & 44,69 & 0,06 & 2,93 & 0,0021 & 0,0181 & 18,0997 \\
$16,500_{\text {cTE11 }}$ & 48,35 & 0,06 & 2,93 & 0,0022 & 0,0188 & 18,7930 \\
$17,750_{\text {cTE11 }}$ & 52,01 & 0,06 & 2,93 & 0,0022 & 0,0195 & 19,4639 \\
$19,000_{\text {cTE11 }}$ & 55,68 & 0,06 & 2,93 & 0,0023 & 0,0201 & 20,1143 \\
$20,250_{\text {cTE11 }}$ & 59,34 & 0,06 & 2,93 & 0,0024 & 0,0207 & 20,7456 \\
\hline
\end{tabular}

Tabla 12. Atenuación para guía de onda circular llena de aire de $3 \mathrm{~cm}$ de radio en el modo TE11. Elaboración propia.

Cuadro 10. Curva de atenuación para la guía de onda de $3 \mathrm{~cm}$ de radio en el modo TE11

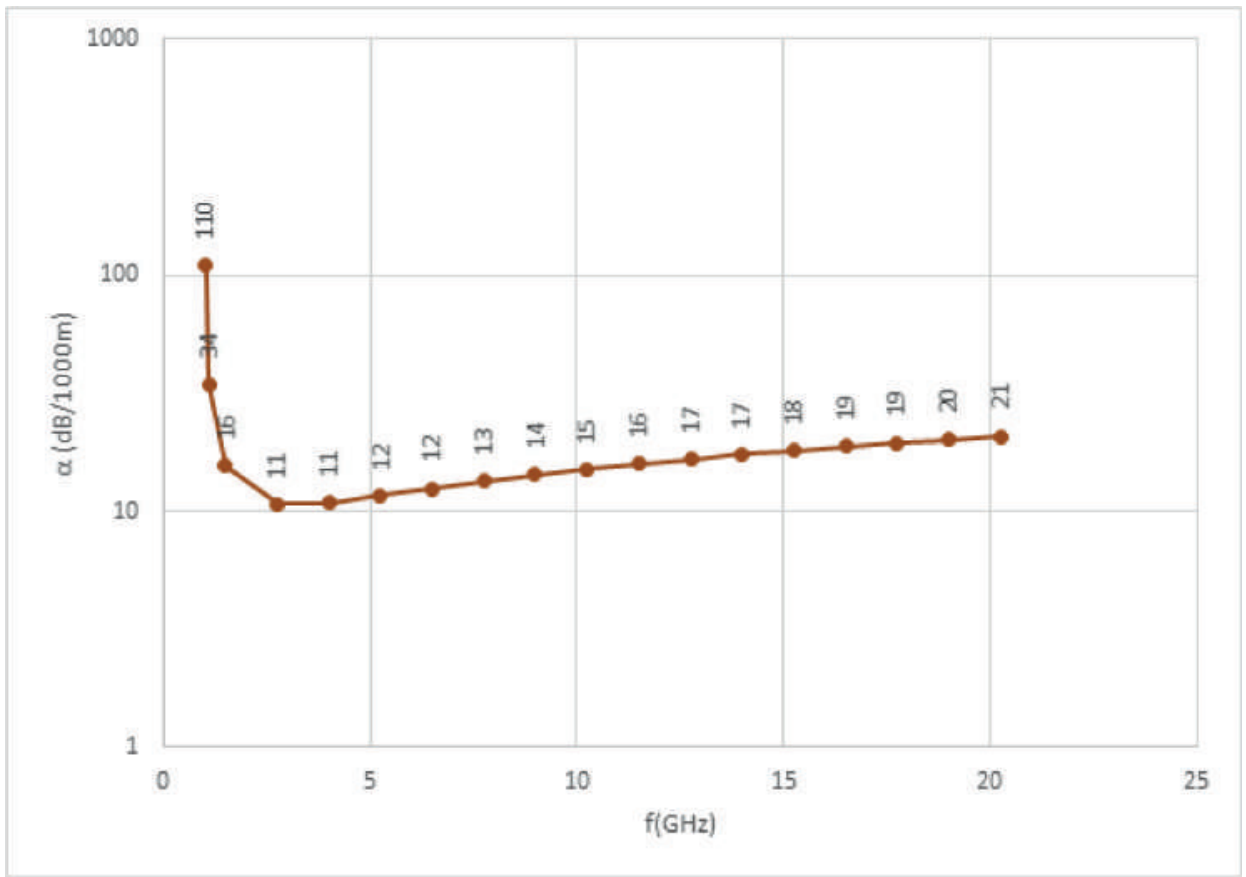

Fuente: Elaboración propia. 


\begin{tabular}{|c|c|c|c|c|c|c|}
\hline & $\begin{array}{c}f \\
(\mathrm{GHz})\end{array}$ & $\begin{array}{c}\mathrm{R}_{\mathrm{s}} \\
\left(\mathrm{Ohmios} / \mathrm{m}^{2}\right)\end{array}$ & $\begin{array}{c}\mathrm{f}_{\mathrm{cTE} 01} \\
(\mathrm{GHz})\end{array}$ & $(\mathrm{Np} / \mathrm{m})$ & $\begin{array}{r}\alpha \\
(\mathrm{GHz})\end{array}$ & $\left(\mathrm{Ohmios} / \mathrm{m}^{2}\right)$ \\
\hline $1,001_{\text {сTE} 21}$ & 4,87 & 0,02 & 4,86 & 0,0635 & 0,5517 & 551,7088 \\
\hline $1,010_{\text {сTE } 21}$ & 4,91 & 0,02 & 4,86 & 0,0201 & 0,1746 & 174,6411 \\
\hline $1,100_{\text {сTЕ } 21}$ & 5,35 & 0,02 & 4,86 & 0,0064 & 0,0560 & 55,9524 \\
\hline $2,000_{\text {cTE11 }}$ & 5,86 & 0,02 & 4,86 & 0,0046 & 0,0398 & 39,8465 \\
\hline 3,250 cTE11 & 9,52 & 0,03 & 4,86 & 0,0027 & 0,0232 & 23,1948 \\
\hline $4,500_{\text {сTE11 }}$ & 13,19 & 0,03 & 4,86 & 0,0025 & 0,0221 & 22,1368 \\
\hline $5,750_{\text {сTE11 }}$ & 16,85 & 0,03 & 4,86 & 0,0026 & 0,0229 & 22,8511 \\
\hline $7,000_{\text {cTE11 }}$ & 20,51 & 0,04 & 4,86 & 0,0028 & 0,0240 & 24,0421 \\
\hline 8,250 cTE11 & 24,18 & 0,04 & 4,86 & 0,0029 & 0,0254 & 25,3812 \\
\hline $9,500_{\text {сTE1 } 1}$ & 27,84 & 0,04 & 4,86 & 0,0031 & 0,0268 & 26,7559 \\
\hline $10,750_{\text {cTE } 11}$ & 31,50 & 0,05 & 4,86 & 0,0032 & 0,0281 & 28,1218 \\
\hline $12,000_{\text {сTE11 }}$ & 35,16 & 0,05 & 4,86 & 0,0034 & 0,0295 & 29,4606 \\
\hline $13,250_{\text {cTE11 }}$ & 38,83 & 0,05 & 4,86 & 0,0035 & 0,0308 & 30,7650 \\
\hline $14,500_{\text {cTE } 11}$ & 42,49 & 0,05 & 4,86 & 0,0037 & 0,0320 & 32,0328 \\
\hline $15,750_{\text {cTE } 11}$ & 46,15 & 0,06 & 4,86 & 0,0038 & 0,0333 & 33,2642 \\
\hline 17,000 сTE11 & 49,82 & 0,06 & 4,86 & 0,0040 & 0,0345 & 34,4604 \\
\hline $18,250_{\text {сTE11 }}$ & 53,48 & 0,06 & 4,86 & 0,0041 & 0,0356 & 35,6230 \\
\hline $19,500_{\text {cTE } 11}$ & 57,14 & 0,06 & 4,86 & 0,0042 & 0,0368 & 36,7541 \\
\hline $20,750_{\text {сTE} 11}$ & 60,80 & 0,06 & 4,86 & 0,0044 & 0,0379 & 37,8554 \\
\hline
\end{tabular}

Tabla 13. Atenuación para guía de onda circular llena de aire de $3 \mathrm{~cm}$ de radio en el modo TE21. Elaboración propia.

CuAdro 11. Curva de atenuación para la guía de onda de $3 \mathrm{~cm}$ de radio en el modo TE21

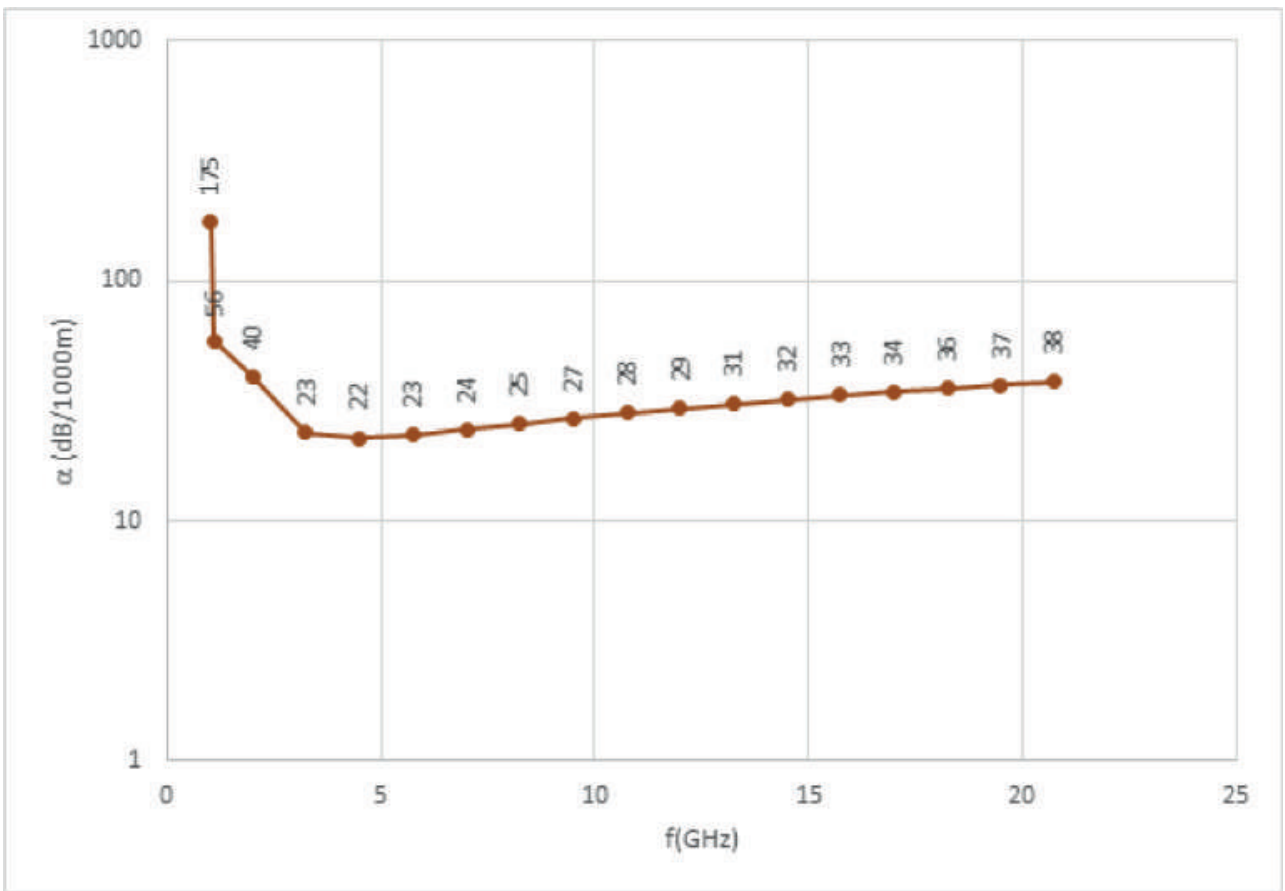

Fuente: Elaboración propia. 


\begin{tabular}{|c|c|c|c|c|c|c|}
\hline & $\begin{array}{c}\mathrm{f} \\
(\mathrm{GHz})\end{array}$ & $\begin{array}{c}\mathrm{R}_{\mathrm{s}} \\
\left(\mathrm{Ohmios} / \mathrm{m}^{2}\right)\end{array}$ & $\begin{array}{c}f_{\text {cTE01 }} \\
(\mathrm{GHz})\end{array}$ & $(\mathrm{Np} / \mathrm{m})$ & $\begin{array}{r}\alpha \\
(\mathrm{GHz})\end{array}$ & $\left(\mathrm{Ohmios} / \mathrm{m}^{2}\right)$ \\
\hline $1,001_{\text {стм01 }}$ & 3,83 & 0,02 & 3,83 & 0,0322 & 0,2800 & 279,9534 \\
\hline $1,010_{\text {стм } 01}$ & 3,87 & 0,02 & 3,83 & 0,0103 & 0,0895 & 89,5246 \\
\hline $1,100_{\text {стм } 01}$ & 4,21 & 0,02 & 3,83 & 0,0036 & 0,0315 & 31,4802 \\
\hline 2,000 сTE11 & 5,86 & 0,02 & 3,83 & 0,0024 & 0,0204 & 20,4321 \\
\hline $3,250_{\text {сTE } 11}$ & 9,52 & 0,03 & 3,83 & 0,0025 & 0,0215 & 21,5405 \\
\hline $4,500_{\text {cTE11 }}$ & 13,19 & 0,03 & 3,83 & 0,0028 & 0,0243 & 24,2537 \\
\hline 5,750 сTE11 & 16,85 & 0,03 & 3,83 & 0,0031 & 0,0269 & 26,9401 \\
\hline $7,000_{\text {сTE11 }}$ & 20,51 & 0,04 & 3,83 & 0,0034 & 0,0295 & 29,4649 \\
\hline $8,250_{\text {сTE1 } 1}$ & 24,18 & 0,04 & 3,83 & 0,0037 & 0,0318 & 31,8273 \\
\hline $9,500_{\text {cTE} 11}$ & 27,84 & 0,04 & 3,83 & 0,0039 & 0,0340 & 34,0460 \\
\hline $10,750_{\text {сTE } 11}$ & 31,50 & 0,05 & 3,83 & 0,0042 & 0,0361 & 36,1405 \\
\hline $12,000_{\text {сTE } 11}$ & 35,16 & 0,05 & 3,83 & 0,0044 & 0,0381 & 38,1275 \\
\hline $13,250_{\text {сTE } 11}$ & 38,83 & 0,05 & 3,83 & 0,0046 & 0,0400 & 40,0211 \\
\hline $14,500_{\text {сTE11 }}$ & 42,49 & 0,05 & 3,83 & 0,0048 & 0,0418 & 41,8325 \\
\hline $15,750_{\text {сTE11 }}$ & 46,15 & 0,06 & 3,83 & 0,0050 & 0,0436 & 43,5712 \\
\hline $17,000_{\text {сTE} 11}$ & 49,82 & 0,06 & 3,83 & 0,0052 & 0,0452 & 45,2450 \\
\hline $18,250_{\text {сTE11 }}$ & 53,48 & 0,06 & 3,83 & 0,0054 & 0,0469 & 46,8605 \\
\hline $19,500_{\text {сTE11 }}$ & 57,14 & 0,06 & 3,83 & 0,0056 & 0,0484 & 48,4233 \\
\hline 20,750 сTE11 & 60,80 & 0,06 & 3,83 & 0,0057 & 0,0499 & 49,9380 \\
\hline
\end{tabular}

Tabla 14. Atenuación para guía de onda circular llena de aire de $3 \mathrm{~cm}$ de radio en el modo TMO1. Elaboración propia.

CuAdro 12. Curva de atenuación para la guía de onda de $3 \mathrm{~cm}$ de radio en el modo TM01

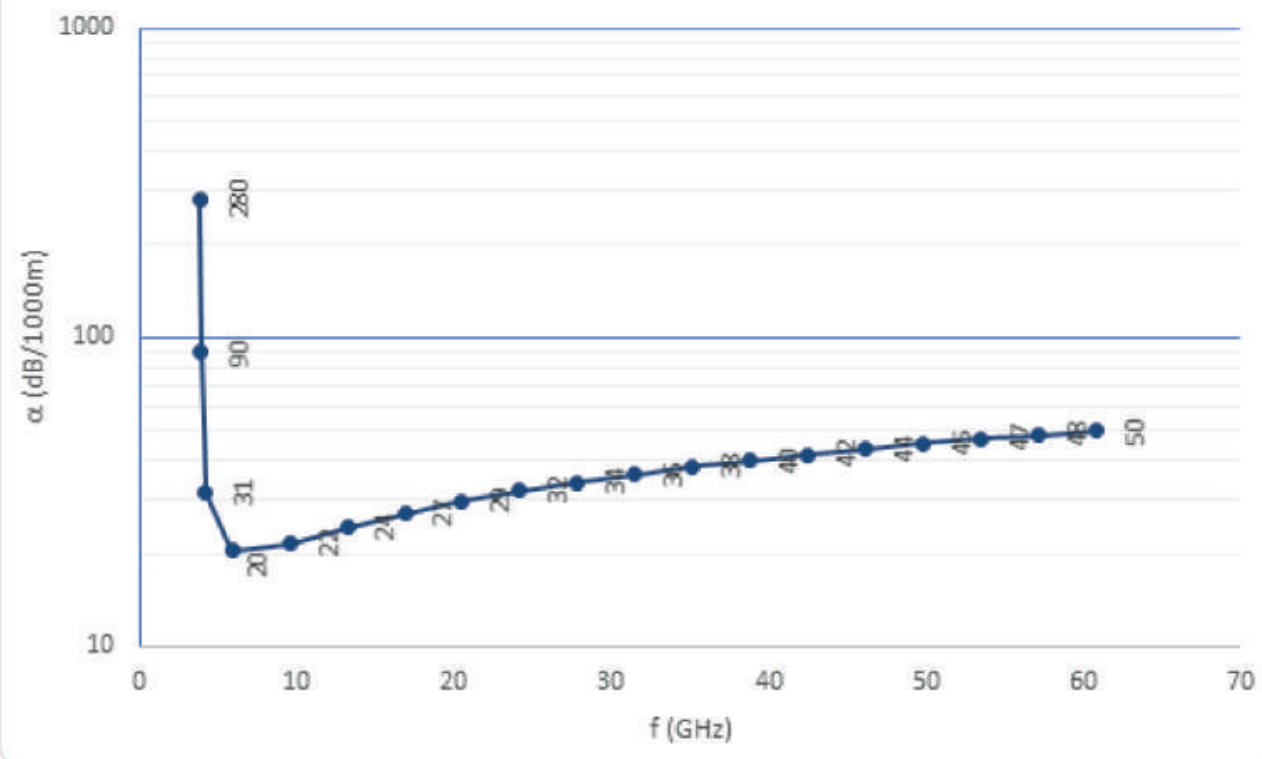

Fuente: Elaboración propia. 


\begin{tabular}{|c|c|c|c|c|c|c|}
\hline & $\begin{array}{c}f \\
(\mathrm{GHz})\end{array}$ & $\begin{array}{c}\mathrm{R}_{\mathrm{s}} \\
\left(\mathrm{Ohmios} / \mathrm{m}^{2}\right)\end{array}$ & $\begin{array}{c}f_{\text {сTE01 }} \\
(\mathrm{GHz})\end{array}$ & $(\mathrm{Np} / \mathrm{m})$ & $\begin{array}{r}\alpha \\
(\mathrm{GHz})\end{array}$ & $\left(\mathrm{Ohmios} / \mathrm{m}^{2}\right)$ \\
\hline $1,001_{\text {стм11 }}$ & 6,10 & 0,02 & 6,10 & 0,0407 & 0,3534 & 353,3774 \\
\hline $1,010_{\text {стМ } 11}$ & 6,16 & 0,02 & 6,10 & 0,0130 & 0,1130 & 113,0044 \\
\hline $1,100_{\text {стМ } 11}$ & 6,71 & 0,02 & 6,10 & 0,0046 & 0,0397 & 39,7366 \\
\hline $3,250_{\text {cTE} 11}$ & 9,52 & 0,03 & 6,10 & 0,0030 & 0,0257 & 25,6800 \\
\hline $4,500_{\text {cTE } 11}$ & 13,19 & 0,03 & 6,10 & 0,0030 & 0,0262 & 26,1772 \\
\hline $5,750_{\text {cTE} 11}$ & 16,85 & 0,03 & 6,10 & 0,0032 & 0,0281 & 28,1439 \\
\hline $7,000_{\text {cTE} 11}$ & 20,51 & 0,04 & 6,10 & 0,0035 & 0,0303 & 30,3183 \\
\hline 8,250 cTE11 & 24,18 & 0,04 & 6,10 & 0,0037 & 0,0325 & 32,4762 \\
\hline $9,500_{\text {cTE} 11}$ & 27,84 & 0,04 & 6,10 & 0,0040 & 0,0346 & 34,5622 \\
\hline 10,750 сTE11 & 31,50 & 0,05 & 6,10 & 0,0042 & 0,0366 & 36,5645 \\
\hline $12,000_{\text {сTE } 11}$ & 35,16 & 0,05 & 6,10 & 0,0044 & 0,0385 & 38,4842 \\
\hline $13,250_{\text {сTE } 11}$ & 38,83 & 0,05 & 6,10 & 0,0046 & 0,0403 & 40,3267 \\
\hline $14,500_{\text {сTE } 11}$ & 42,49 & 0,05 & 6,10 & 0,0048 & 0,0421 & 42,0983 \\
\hline 15,750 сTE11 & 46,15 & 0,06 & 6,10 & 0,0050 & 0,0438 & 43,8052 \\
\hline $17,000_{\text {сTE1 } 1}$ & 49,82 & 0,06 & 6,10 & 0,0052 & 0,0455 & 45,4531 \\
\hline $18,250_{\text {cTE } 11}$ & 53,48 & 0,06 & 6,10 & 0,0054 & 0,0470 & 47,0472 \\
\hline $19,500_{\text {сTE } 11}$ & 57,14 & 0,06 & 6,10 & 0,0056 & 0,0486 & 48,5920 \\
\hline 20,750 СТE 11 & 60,80 & 0,06 & 6,10 & 0,0058 & 0,0501 & 50,0916 \\
\hline
\end{tabular}

Tabla 15. Atenuación para guía de onda circular llena de aire de $3 \mathrm{~cm}$ de radio en el modo TM11. Elaboración propia.

CuAdro 13. Curva de atenuación para la guía de onda de $3 \mathrm{~cm}$ de radio en el modo TM11

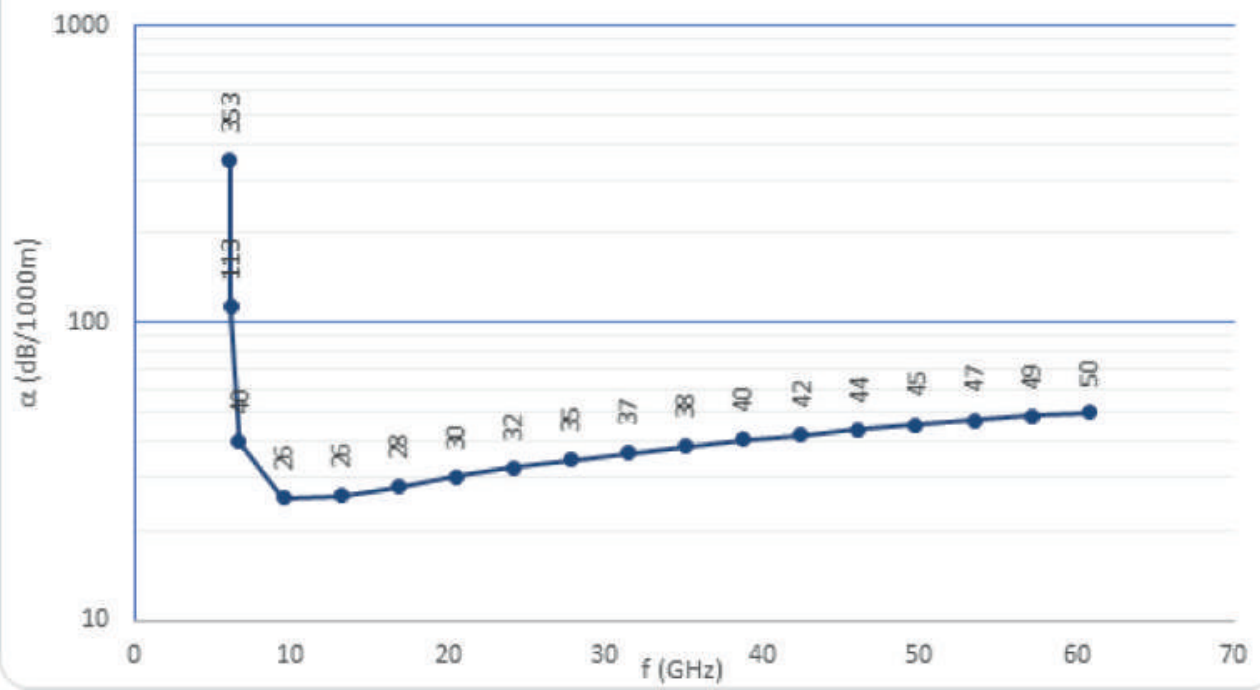

Fuente: Elaboración propia. 


\begin{tabular}{|c|c|c|c|c|c|c|}
\hline $\mathrm{f} / \mathrm{f}_{\mathrm{cTE} 11}$ & $\begin{array}{c}\alpha_{\mathrm{TE} 01} \\
(\mathrm{~dB} / 1000 \mathrm{~m})\end{array}$ & $\begin{array}{c}\alpha_{\mathrm{TE} 02} \\
(\mathrm{~dB} / 1000 \mathrm{~m})\end{array}$ & $\begin{array}{c}\alpha_{\text {TE11 }} \\
(\mathrm{dB} / 1000 \mathrm{~m})\end{array}$ & $\begin{array}{c}\alpha_{\text {TE21 }} \\
(\mathrm{dB} / 1000 \mathrm{~m})\end{array}$ & $\begin{array}{c}\alpha_{\mathrm{TM} 01} \\
(\mathrm{~dB} / 1000 \mathrm{~m})\end{array}$ & $\begin{array}{c}\alpha_{\text {TM11 }} \\
(\mathrm{dB} / 1000 \mathrm{~m})\end{array}$ \\
\hline 2.00 & & & 11.9422 & 39.8465 & 20.4321 & \\
\hline 2.50 & & & 10.9175 & 27.5390 & 20.2887 & \\
\hline 3.00 & 12.6619 & & 10.6433 & 24.0279 & 21.0504 & 26.3110 \\
\hline 3.50 & 9.0012 & & 10.6805 & 22.6726 & 22.0628 & 25.4584 \\
\hline 4.00 & 6.9362 & & 10.8684 & 22.1888 & 23.1512 & 25.6234 \\
\hline 4.50 & 5.5989 & & 11.1356 & 22.1368 & 24.2537 & 26.1772 \\
\hline 5.00 & 4.6614 & 21.9430 & 11.4462 & 22.3238 & 25.3451 & 26.9065 \\
\hline 5.50 & 3.9689 & 17.0775 & 11.7805 & 22.6520 & 26.4148 & 27.7202 \\
\hline 6.00 & 3.4377 & 13.9921 & 12.1274 & 23.0672 & 27.4586 & 28.5740 \\
\hline 6.50 & 3.0184 & 11.8317 & 12.4800 & 23.5373 & 28.4752 & 29.4444 \\
\hline 7.00 & 2.6799 & 10.2246 & 12.8342 & 24.0421 & 29.4649 & 30.3183 \\
\hline 7.50 & 2.4014 & 8.9790 & 13.1873 & 24.5687 & 30.4284 & 31.1881 \\
\hline 8.00 & 2.1689 & 7.9840 & 13.5378 & 25.1084 & 31.3669 & 32.0495 \\
\hline 8.50 & 1.9722 & 7.1709 & 13.8845 & 25.6553 & 32.2819 & 32.8998 \\
\hline 9.00 & 1.8040 & 6.4941 & 14.2268 & 26.2054 & 33.1745 & 33.7376 \\
\hline 9.50 & 1.6587 & 5.9223 & 14.5644 & 26.7559 & 34.0460 & 34.5622 \\
\hline 10.00 & 1.5321 & 5.4332 & 14.8970 & 27.3047 & 34.8977 & 35.3732 \\
\hline 10.50 & 1.4210 & 5.0103 & 15.2246 & 27.8504 & 35.7307 & 36.1708 \\
\hline 11.00 & 1.3228 & 4.6415 & 15.5472 & 28.3920 & 36.5460 & 36.9549 \\
\hline 11.50 & 1.2355 & 4.3171 & 15.8648 & 28.9289 & 37.3447 & 37.7259 \\
\hline 12.00 & 1.1575 & 4.0299 & 16.1775 & 29.4606 & 38.1275 & 38.4842 \\
\hline 12.50 & 1.0874 & 3.7740 & 16.4855 & 29.9866 & 38.8955 & 39.2301 \\
\hline 13.00 & 1.0242 & 3.5447 & 16.7887 & 30.5070 & 39.6493 & 39.9641 \\
\hline 13.50 & 0.9669 & 3.3382 & 17.0875 & 31.0215 & 40.3896 & 40.6865 \\
\hline 14.00 & 0.9148 & 3.1514 & 17.3819 & 31.5301 & 41.1171 & 41.3977 \\
\hline 14.50 & 0.8672 & 2.9818 & 17.6721 & 32.0328 & 41.8325 & 42.0983 \\
\hline 15.00 & 0.8237 & 2.8271 & 17.9581 & 32.5297 & 42.5362 & 42.7885 \\
\hline 15.50 & 0.7836 & 2.6855 & 18.2402 & 33.0208 & 43.2288 & 43.4687 \\
\hline 16.00 & 0.7468 & 2.5556 & 18.5185 & 33.5062 & 43.9109 & 44.1393 \\
\hline 16.50 & 0.7127 & 2.4359 & 18.7930 & 33.9860 & 44.5828 & 44.8007 \\
\hline 17.00 & 0.6812 & 2.3255 & 19.0640 & 34.4604 & 45.2450 & 45.4531 \\
\hline 17.50 & 0.6519 & 2.2232 & 19.3315 & 34.9294 & 45.8978 & 46.0969 \\
\hline 18.00 & 0.6247 & 2.1283 & 19.5956 & 35.3931 & 46.5418 & 46.7325 \\
\hline 18.50 & 0.5993 & 2.0401 & 19.8565 & 35.8517 & 47.1771 & 47.3600 \\
\hline 19.00 & 0.5756 & 1.9578 & 20.1143 & 36.3053 & 47.8042 & 47.9798 \\
\hline 19.50 & 0.5535 & 1.8810 & 20.3690 & 36.7541 & 48.4233 & 48.5920 \\
\hline 20.00 & 0.5327 & 1.8091 & 20.6208 & 37.1980 & 49.0347 & 49.1971 \\
\hline
\end{tabular}

Tabla 16. Comparación entre las atenuaciones de diversos modos de transmisión TE y TM para guía de onda circular llena de aire de $3 \mathrm{~cm}$ de radio. Elaboración propia. 
Cuadro 14. Curva de atenuación para la guía de onda de $3 \mathrm{~cm}$ de radio

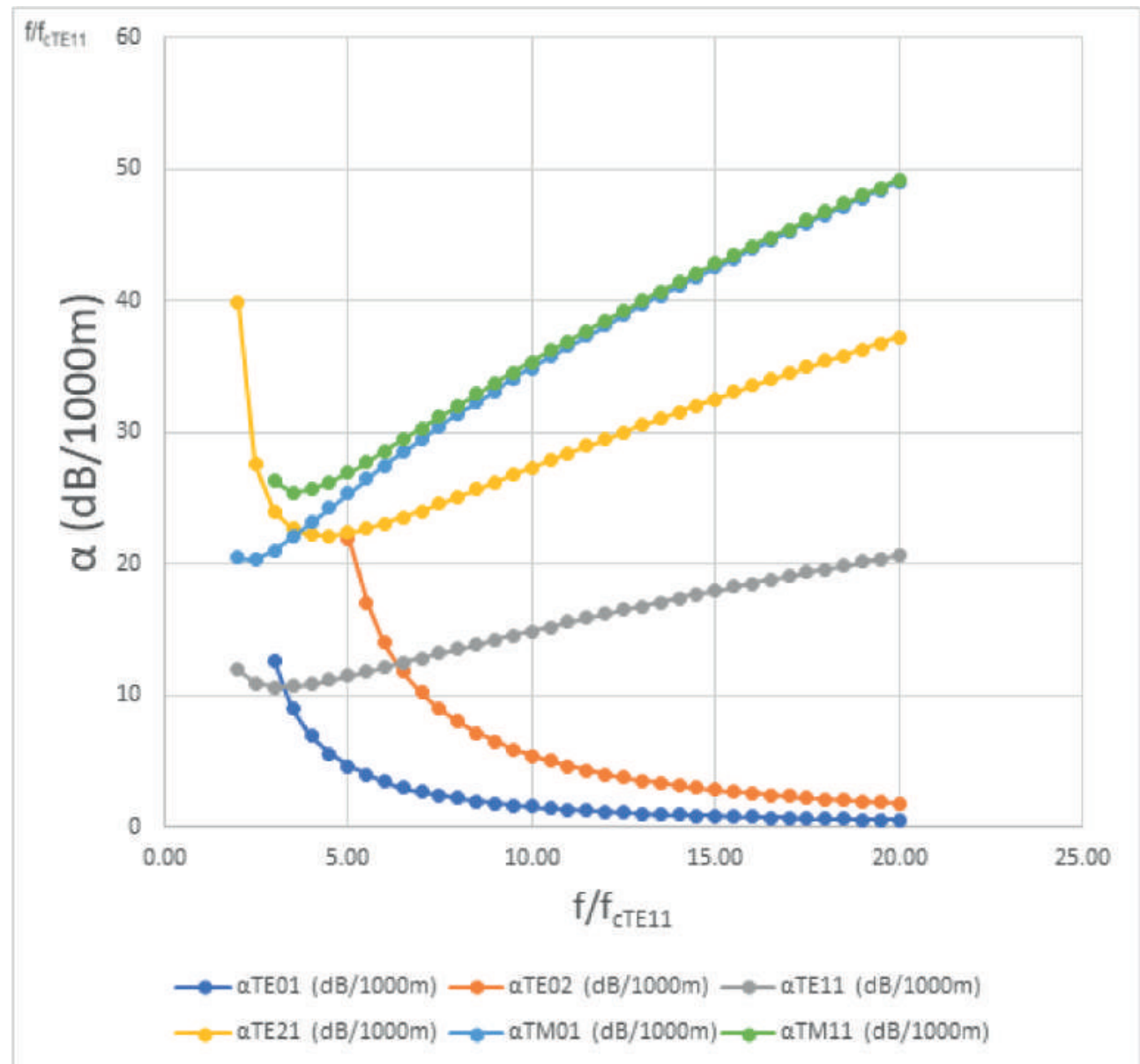

Fuente: Elaboración propia.

\section{DISCUSIÓN}

Al comparar las gráficas resultantes de los cálculos mostradas por Balanis [4], en todos los casos hay coincidencia, a excepción de la guía de onda circular TM21.

\section{REFERENCIAS BIBLIOGRÁFICAS}

[1] J.D. Kraus y D.A. Fleis, Electromagnetismo: con aplicaciones. Ciudad de México, México: McGrawHill Interamericana, 2000.

[2] M. Sadiku, Elementos de electromagnetismo. Oxford University Press Inc, Nueva York, USA: Editorial Alfa Omega para América Latina, 2000.

[3] R E Collin, Foundations for microwave engineering. Wiley-IEEE Press, Nueva York, USA: Editorial John Wiley \& Sons, Inc., 2001. 
[4] C. A. Balanis, "Circular waveguides 1». Int. J. Electron 1996.5 (1996), pp. 551-564.

[5] C. S. Lee, S. W. Lee, and S. L. Chuang, "Plot of modal field distribution in rectangular and circular waveguides, IEEE Trans. Microwave Theory Tech. MTT-33(3):271-274 (marzo 1985).

[6] S. Ramo, J. R. Whinnery, and T. Van Duzer, Fields and Waves in Communication Electronics, Nueva York, USA: Wiley, 1965.

[7] R. Gómez Martín, Campo Electromagnético: Propagación y Radiación. Granada, España: Univ. de Granada, 1984 [En línea]. Disponible en: http://maxwell.ugr.es/innov/visua0506/librorgomez/librorgomez.htm 